Discrete Comput Geom 26:465-492 (2001)

DOI: $10.1007 / \mathrm{s} 00454-001-0043-\mathrm{x}$

\title{
On the Complexity of Arrangements of Circles in the Plane*
}

\author{
N. Alon, ${ }^{1}$ H. Last, ${ }^{2}$ R. Pinchasi, ${ }^{2}$ and M. Sharir ${ }^{1,3}$ \\ ${ }^{1}$ School of Mathematical Sciences, Tel Aviv University, \\ Tel Aviv 69978, Israel \\ \{noga,sharir\}@math.tau.ac.il \\ ${ }^{2}$ Institute of Mathematics, Hebrew University, \\ Givat Ram, Jerusalem, Israel \\ \{hagitl,room\}@math.huji.ac.il \\ ${ }^{3}$ Courant Institute of Mathematical Sciences, New York University, \\ New York, NY 10012, USA
}

\begin{abstract}
Continuing and extending the analysis in a previous paper [15], we establish several combinatorial results on the complexity of arrangements of circles in the plane. The main results are a collection of partial solutions to the conjecture that (a) any arrangement of unit circles with at least one intersecting pair has a vertex incident to at most three circles, and (b) any arrangement of circles of arbitrary radii with at least one intersecting pair has a vertex incident to at most three circles, under appropriate assumptions on the number of intersecting pairs of circles (see below for a more precise statement).
\end{abstract}

\section{Introduction}

In this paper we study the combinatorial complexity of arrangements of circles in the plane. The main motivation for our study is the following conjecture, whose parts (a) and (b) have been posed by A. Bezdek for the case of unit circles [5] (see also [3] and [4] for related conjectures), and parts (c) and (d) are extensions to the case of arbitrary circles. This conjecture extends the classical Sylvester-Gallai problem (see [13]) to the case of circles.

\footnotetext{
* Work by Noga Alon has been supported by a grant from the U.S.-Israeli Binational Science Foundation, by a grant from the Israel Science Foundation and by the Hermann Minkowski-MINERVA Center for Geometry at Tel Aviv University. Work by Micha Sharir has been supported by NSF Grant CCR-97-32101, by a grant from the U.S.-Israeli Binational Science Foundation, by a grant from the Israeli Academy of Sciences for a Center of Excellence in Geometric Computing at Tel Aviv University, by the ESPRIT IV LTR Project No. 21957 (CGAL), and by the Hermann Minkowski-MINERVA Center for Geometry at Tel Aviv University.
} 


\section{Conjecture 1.1.}

(a) Let $\mathcal{C}$ be a finite family of unit circles in the plane, at least two of which intersect. Then there exists an intersection point that is incident to at most three circles of $\mathcal{C}$.

(b) Same as (a), under the additional assumption that every pair of circles of $\mathcal{C}$ intersect.

(c) Let $\mathcal{C}$ be a finite family of arbitrary circles in the plane, such that at least two of them intersect and the number $P$ of intersecting pairs satisfies $P \geq \beta|\mathcal{C}| q$, where $\beta$ is a sufficiently large constant and where $q$ is the maximum size of a pencil of $\mathcal{C}$, namely, a subset all of whose elements are incident to the same pair of points. Then there exists an intersection point that is incident to at most three circles of $\mathcal{C}$.

(d) Let $\mathcal{C}$ be a finite family of arbitrary circles in the plane, such that every pair of circles in $\mathcal{C}$ intersect, and such that $\mathcal{C}$ is not a single pencil. Then there exists an intersection point that is incident to at most three circles of $\mathcal{C}$.

Conjecture 1.1(b) has been proven in a preceding paper of Pinchasi [15]. In fact, it has been shown there that if $\mathcal{C}$ consists of at least five pairwise-intersecting unit circles, then there exists an intersection point incident to just two circles. That paper also gives an example of four pairwise-intersecting unit circles where every intersection point is incident to three circles. This is done as follows. Choose three points on one circle, $a$, such that they form an acute triangle. Let $b, c$ and $d$ be the three unit circles different from $a$ and passing through pairs of these three points. It is not hard to show that $b, c$ and $d$ pass through a common point.

This example can be extended to yield a family of any number of unit circles (not every pair of which intersect) where every intersection point is incident to three circles, and where the intersection graph of the circles is connected. For example, one can build larger "tree-like" examples by repeatedly adding triplets of unit circles using the following procedure: Consider first that arc of, say, $b$ which lies outside of $a, c$ and $d$, then choose three points close to each other on this arc, so that the three unit circles $e, f$ and $g$, which are different from $b$ and pass through pairs of the selected points, are disjoint from $a, c$ and $d$. Notice that every intersection point determined by the family $\{a, b, \ldots, g\}$ has degree 3 .

Another simple construction proceeds as follows. Let $R$ be a rhombus of edge length 2 and with angles $>60^{\circ}$ and consider a simple connected cluster of congruent copies of $R$ glued to each other in an edge-to-edge manner. The unit circles which are centered at the vertices and at the midpoints of these rhombi form a family of unit circles where each intersection point has degree 3 . One can also add triplets of unit circles to this arrangement, in the same way described above, to obtain more complicated examples with the same property.

Finally, we note that it is much easier to establish the existence of a vertex incident to no more than five circles (in all versions of the conjecture). Such an argument is given in [7]. However, reducing "five" to "four" (let alone "three," as in the conjecture) is not easy.

In this paper we prove various special cases of the other three parts of Conjecture 1.1. 
We first study Conjecture 1.1(d), and prove it in the case that $n$, the size of $\mathcal{C}$, is sufficiently large (see Theorem 4.1). We then tackle Conjecture 1.1(a), and prove it in the case that $n$ is sufficiently large and the number of pairs of intersecting circles in $\mathcal{C}$ is at least $\beta n$, for an appropriate absolute positive constant $\beta$ (see Theorem 4.5). Finally, we give a proof of Conjecture 1.1(c), under the stronger assumption that $P \geq \beta|\mathcal{C}|\left(q+|\mathcal{C}|^{1 / 3}\right)$ for an appropriate absolute constant $\beta$ (see Theorem 4.8). We also note that part (c) of the conjecture fails if $P \leq|\mathcal{C}| q / 2$ (see the remark at the end of Section 4).

Someof the technical tools that we develop for our solutions are, in our opinion, of independent interest. The main set of tools deals with faces of degree 2 in the arrangement $\mathcal{A}(\mathcal{C})$ of $\mathcal{C}$. With the possible exception of the unbounded face, these faces are either "lenses" (contained in the interiors of the two incident circles) or "lunes" (contained in the interior of one incident circle and in the exterior of the other). We derive various upper bounds for the number of these faces: In case (b) it was shown in [15] that the number of lunes is at most three ${ }^{1}$ and the number of lenses is at most $n$. In case (d) we show that the number of lunes is at most $2 n-2$ and the number of lenses is at most $18 n$. In case (a) we show that the number of lenses is at most $O\left(n^{4 / 3} \log n\right)$ (and the number of lunes is at most $n$ ). In case (c) we show that the number of lenses and lunes is $O\left(n^{3 / 2+\varepsilon}\right)$, for any $\varepsilon>0$ (where the constant of proportionality depends on $\varepsilon$ ).

The study of lenses and lunes has also been followed in [6], for similar goals. It is also reminiscent of the study of lenses in arrangements of "pseudo-parabolas" by Tamaki and Tokuyama [20].

Another collection of results that may be of independent interest relates the number $V$ of vertices in an arrangement of circles and the number $P$ of intersecting pairs of circles. Specifically, we first show that in an arrangement of unit circles one always has $V=\Omega(P)$ (see Theorem 5.1). The same result also holds for arrangements of general circles, provided that $P \geq \beta|\mathcal{C}|\left(q+|\mathcal{C}|^{1 / 3}\right)$, for some sufficiently large constant $\beta$ (see Theorem 5.4).

The paper is organized as follows. After introducing some notations in Section 2, we prove in Section 3 the above-mentioned upper bounds on the number of lunes and lenses in the various kinds of arrangements of circles under consideration.

We then show, in Section 4, the existence of an intersection point incident to at most three circles, in the various arrangements of circles under consideration, under appropriate additional conditions, as mentioned above.

Finally, in Section 5 we exploit the machinery developed in the paper and prove, for an arrangement of $n$ unit or arbitrary circles, that $V=\Omega(P)$ (for arbitrary circles, under the additional assumptions made above).

\section{Preliminaries}

Throughout this paper $\mathcal{C}$ denotes a finite family of circles in the Euclidean plane.

We usually denote a circle by the letter $C$, possibly with some modifier (subscript or

\footnotetext{
${ }^{1}$ Actually, this was shown under the additional assumption that $\mathcal{A}(\mathcal{C})$ does not contain any vertex incident to just two circles. Nevertheless, recent unpublished work by Last and Pinchasi show that this is true for any arrangement of pairwise-intersecting unit circles.
} 


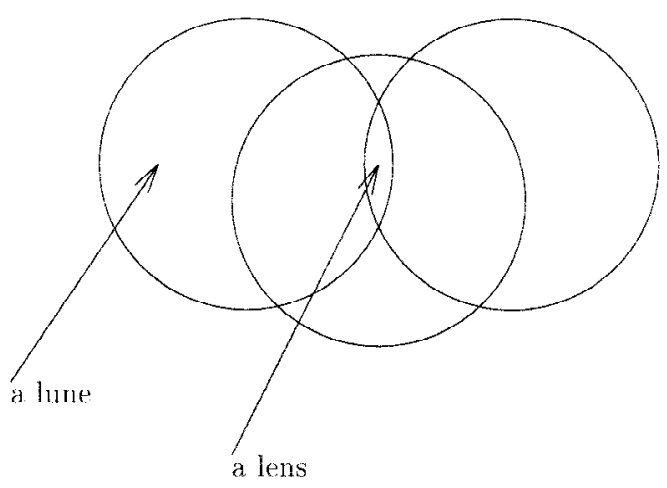

Fig. 1. A lens and a lune.

superscript). The closed disk bounded by that circle is denoted by $D$, and its center by $o$, with the same modifier.

Definition 2.1. Let $\mathcal{C}$ be a family of circles in the plane. Let $C_{1}, C_{2}$ be two circles in $\mathcal{C}$ that intersect at two distinct points, $A$ and $B$. We call $D_{1} \cap D_{2}$ a lens if no circle in $\mathcal{C} \backslash\left\{C_{1}, C_{2}\right\}$ meets $\left(D_{1} \cap D_{2}\right) \backslash\{A, B\}$. We then say that both $C_{1}$ and $C_{2}$ support that lens (see Fig. 1).

We call $D_{1} \backslash$ int $D_{2}$ a lune if no circle in $\mathcal{C} \backslash\left\{C_{1}, C_{2}\right\}$ meets $\left(D_{1} \backslash\right.$ int $\left.D_{2}\right) \backslash\{A, B\}$. We then call $C_{1} \backslash$ int $D_{2}$ the longer arc of the lune and say that $C_{1}$ supports the longer arc of that lune.

Whenever we refer to two intersecting circles we mean two circles that either intersect at two distinct points or are tangent.

For a circle $C$ and points $a, b$ on $C$ which are not antipodal, we denote by $\widehat{C}(a b)$ the closed smaller arc of $C$ delimited by $a$ and $b$.

For two distinct points $a$ and $b$ in the plane, we denote by $\overline{a b}$ the line through $a$ and $b$. We denote by $\overrightarrow{a b}$ the closed ray that emanates from $a$ and contains $b$. The closed line segment between $a$ and $b$ is denoted by $[a b]$.

Let $p, q, r$ be three noncollinear distinct points in the plane. We denote by $\angle p q r$ the closed convex region bounded by the rays $\overrightarrow{q p}$ and $\overrightarrow{q r}$. The angular measure of $\angle p q r$ is denoted by $\measuredangle p q r$. Therefore $0<\measuredangle p q r<\pi$.

In a previous paper [15] and in more recent unpublished work by Last and Pinchasi, the following result is proved.

Theorem 2.2 [15]. A family of $n$ pairwise-intersecting unit circles in the plane determines at most three lunes and at most $n$ lenses.

In the following section we extend this result for more general arrangements of circles. 


\section{Bounding the Number of Lunes and Lenses in Arrangements of Circles}

In this section we obtain upper bounds on the number of lunes and lenses in various types of arrangements of circles: arrangements of pairwise-intersecting circles, arrangements of unit circles, and arrangements of arbitrary circles (in the two latter cases, not every pair of circles is intersecting). These results, besides being of independent interest, are needed for showing that such arrangements, under additional assumptions, must contain vertices incident to at most three circles.

\subsection{The Number of Lunes in a Family of Pairwise-Intersecting Circles}

Inthis subsection we prove the following theorem.

Theorem 3.1. A family of $n$ pairwise-intersecting circles in the plane determines at most $2 n-2$ lunes.

Definition 3.2. Let $\mathcal{C}$ be a family of circles. We say that $\mathcal{C}$ is a pencil if either there are two distinct points that belong to every circle in $\mathcal{C}$, or the circles in $\mathcal{C}$ are pairwise tangent at a common point $P$. We sometimes refer to the latter case as a degenerate pencil.

Observe that if $\mathcal{C}$ is a nondegenerate pencil, then it determines exactly $2 n-2$ lunes. This shows that Theorem 3.1 is tight in the worst case.

Let $\mathcal{C}$ be a family of $n$ pairwise-intersecting circles in the plane. Define a graph $G$ whose vertices are the centers of the circles in $\mathcal{C}$, and whose edges connect pairs of centers whose associated circles support the same lune. By drawing the edges of $G$ as straight segments, we obtain a plane embedding of this graph.

Observe that unless $\mathcal{C}$ is a pencil, there are no multiple edges in $G$. Indeed, suppose to the contrary that there exist $C_{1}, C_{2} \in \mathcal{C}$ such that both $L_{1}=D_{1} \backslash$ int $D_{2}$ and $L_{2}=$ $D_{2} \backslash$ int $D_{1}$ are lunes. Denote the intersection points of $C_{1}$ and $C_{2}$ by $a, b$. These points partition $C_{1}$ into two arcs, one of which is the outer arc of $L_{1}$ and the other is the inner $\operatorname{arc}$ of $L_{2}$. Hence neither of the relative interiors of these arcs meets another circle, so all circles in $\mathcal{C}$ pass through $a$ and $b$.

Lemma 3.3. $G$ is planar.

Proof. We will show that the plane embedding of $G$ defined above has no pair of crossing edges. This will be a special case of the following more general lemma, which will be needed when we later consider families that are not pairwise intersecting.

Lemma 3.4. Let $C_{1}, C_{2}, C_{3}, C_{4}$ be four distinct circles, such that both $L_{1}=D_{2} \backslash$ int $D_{1}$ and $L_{2}=D_{4} \backslash$ int $D_{3}$ are lunes, and such that all pairs of these circles, with the possible exception of the pair $\left(C_{2}, C_{4}\right)$, are intersecting. Then the line segments $\left[o_{1} o_{2}\right]$ and $\left[o_{3} O_{4}\right]$ do not intersect. 


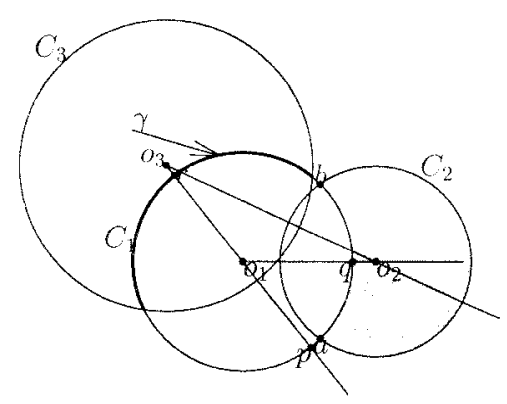

Fig. 2. Case 1 in the proof of Lemma 3.4.

Remark. The proof of Lemma 3.4 applies also to the case where some of the intersecting circles may be tangent to each other. When reading the proof below, the reader should keep in mind that the two points of intersection of a pair of circles may coincide.

Proof of Lemma 3.4. We need the following simple observation:

Observation 3.5. Let $C_{1}$ and $C_{2}$ be two intersecting circles, and denote by $p$ the center of the arc $C_{1} \cap D_{2}$. Then $o_{2}$ lies on the ray $\overrightarrow{o_{1} p}$.

Since $L_{1}$ is a lune, either $D_{3}$ contains $L_{1}$ or $D_{3}$ is disjoint from the interior of $L_{1}$; the same two possibilities hold for $D_{4}$. We consider the following subcases:

Case 1: $D_{3}$ is disjoint from the interior of $L_{1}$. We have to show that $o_{4}$ does not lie inside the convex unbounded region $K$ that is bounded by the rays $\overrightarrow{O_{3} O_{1}}, \overrightarrow{o_{3} O_{2}}$ and by the line segment $\left[o_{1} o_{2}\right]$. Denote by $a, b$ the intersection points of $C_{1}$ and $C_{2}$. Denote by $p$ the intersection point of $\overrightarrow{o_{3} O_{1}}$ with $C_{1}$ that lies outside $D_{3}$. The point $p$ is the farthest point from $o_{3}$ on $C_{1}$ (see Fig. 2). It suffices to show that $o_{4}$ is not inside $\angle o_{2} o_{1} p$.

Let $\gamma$ be the arc $C_{1} \cap D_{3}$. Clearly, $p \notin \gamma$. Let $q$ be the midpoint of the $\operatorname{arc} C_{1} \cap D_{2}$; it is the intersection point of $\overrightarrow{o_{1} o_{2}}$ with $C_{1}$. Since $D_{3}$ is disjoint from the interior of $L_{1}$, we have $q \notin \gamma$. Denote by $\delta$ the arc $C_{1} \cap D_{4}$. Observe that $\delta \subseteq \gamma$, for otherwise $C_{1}$ would intersect the interior of $D_{4} \backslash$ int $D_{3}$, contradicting the assumption that $L_{2}$ is a lune. Hence, by Observation 3.5, $o_{4}$ is on a ray that emanates from $o_{1}$ and crosses $\gamma$.

We claim that $\gamma$, and thus $\delta$ too, is disjoint from $\widehat{C}_{1}(p q)$. To show this, denote by $r$ the midpoint of $\gamma$; clearly, $r$ is the point on $C_{1}$ antipodal to $p$. Recall that both $p$ and $q$ do not lie in $\gamma$. Therefore, if $\gamma \cap \widehat{C}_{1}(p q) \neq \emptyset$, then $\gamma \subseteq \widehat{C}_{1}(p q)$. This however is impossible, since $p$ and $r$ are antipodal points on $C_{1}$ and thus cannot both lie in $\widehat{C}_{1}(p q)$.

Hence, $o_{4}$ does not lie on any of the rays that emanate from $o_{1}$ and cross $\widehat{C}_{1}(p q)$, i.e, $o_{4} \notin \angle o_{2} o_{1} p$.

Case 2: $D_{3} \supset L_{1}$ and $D_{4}$ is disjoint from the interior of $L_{1}$. Rotate the plane so that the line $\overline{o_{1} O_{2}}$ becomes horizontal, and $o_{2}$ is to the right of $o_{1}$. Without loss of generality, assume that $o_{3}$ is in the closed halfplane above $\overline{o_{1} O_{2}}$. We have to show that $o_{4}$ does not lie inside the convex unbounded region $K$ that is bounded by the rays $\overrightarrow{o_{3} o_{1}}, \overrightarrow{o_{3} o_{2}}$ and by 


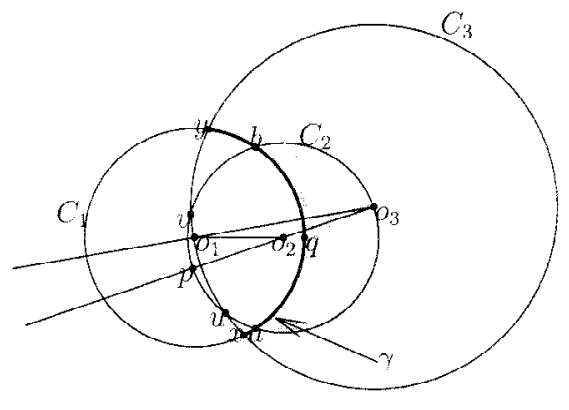

Fig. 3. Case 2 in the proof of Lemma 3.4.

the line segment $\left[o_{1} o_{2}\right]$. Denote by $a, b$ the intersection points of $C_{1}$ and $C_{2}$, so that $a$ is below $\overrightarrow{o_{1} O_{2}}$. Denote by $p$ the intersection point of $\overrightarrow{o_{3} o_{2}}$ with $C_{2}$ that lies outside $D_{3}$; this point is farthest from $o_{3}$ along $C_{2}$.

Denote by $u$ and $v$ the intersection points of $C_{3}$ and $C_{2}$, so that $a, u, v, b$ are in clockwise order along $C_{2}$ (see Fig. 3).

Denote by $\delta$ the arc $C_{1} \cap D_{4}$. Denote by $x, y$ the intersection points of $C_{3}$ and $C_{1}$, so that $x, u, v, y$ are in clockwise order along $C_{3}$ (by assumption, both $u, v$ lie inside $C_{1}$, so no interleaving of $x, y$ with $u, v$ is possible). Denote by $\gamma$ the $\operatorname{arc} C_{1} \cap D_{3}$. The points $x$ and $y$ are the endpoints of $\gamma$. Clearly, $\delta \subseteq \gamma$, for otherwise, arguing as above, $C_{1}$ would intersect the interior of $D_{4} \backslash D_{3}$, contradicting the assumption that $L_{2}$ is a lune. Denote by $q \in C_{1}$ the intersection point of $\overrightarrow{o_{1} o_{2}}$ with $C_{1}$; this is the midpoint of the $\operatorname{arc} C_{1} \cap D_{2}$.

Assume to the contrary that $o_{4}$ lies inside the region $K$. Let $r$ be the midpoint of $\delta$. Since $o_{4}$ is below or on $\overline{o_{1} O_{2}}$, we conclude, by Observation 3.5, that $r$ is also below or on $\overline{o_{1} O_{2}}$. Therefore, $r \in \widehat{C}_{1}(x q)$.

Since we assume that $D_{4}$ is disjoint from the interior of $L_{1}$, we have $r \in \widehat{C}_{1}(x a)$ and also $\delta \subseteq \widehat{C}_{1}(x a)$.

We next claim that $D_{4}$ cannot contain any of the points $a, u, x$ as an interior point. Indeed, $a$ cannot be interior to $D_{4}$, for otherwise $D_{4}$ would intersect the interior of $L_{1}$, which is impossible. If $x$ were interior to $D_{4}$, then $D_{4}$ would intersect the interior of the arc $C_{1} \backslash D_{3}$. Therefore, $C_{1}$ would intersect the interior of $D_{4} \backslash D_{3}$, contradicting the assumption that $L_{2}$ is a lune. Finally, if $u$ were interior to $D_{4}$, then $D_{4}$ would intersect the interior of the arc $C_{2} \backslash D_{3}$. In this case $C_{2}$ would intersect the interior of $D_{4} \backslash D_{3}$, contradicting the assumption that $L_{2}$ is a lune.

We may also assume that $C_{3} \cap D_{4}$, which is the inner arc of $L_{2}$, is contained in $D_{1}$. Otherwise, $D_{1}$ would be disjoint from $L_{2}$, so we could apply Case 1 , switching the roles of $L_{1}$ and $L_{2}$.

Let $e \in D_{1}$ be one of the endpoints of the arc $C_{3} \cap D_{4}$. Denote by $\Delta$ the region bounded by $\widehat{C}_{1}(x a), \widehat{C_{2}}(a u), \widehat{C_{3}}(u x)$.

From the convexity of $D_{4}$, the line segment $[e r]$ is contained in $D_{4}$. It intersects the boundary of $\Delta$ at $r$ and at another point $f$ which lies either on $\widehat{C}_{3}(u x)$ or on $\widehat{C}_{2}(a u)$ (because $e \in \operatorname{int} D_{4}$ ). 


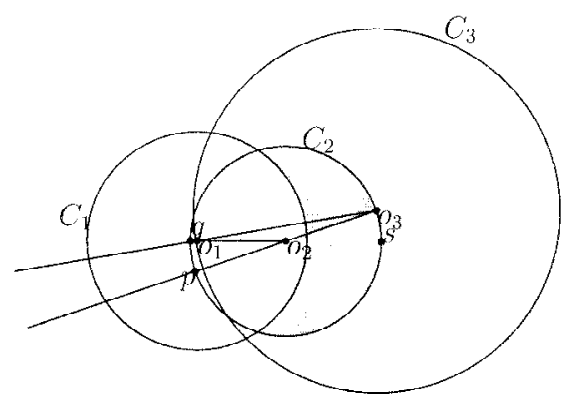

Fig. 4. Case 3 in the proof of Lemma 3.4.

Assume first that $f \in \widehat{C_{3}}(u x)$. By the preceding claim, we have $C_{3} \cap D_{4} \subset \widehat{C_{3}}(u x)$. By Observation 3.5, $o_{4}$ lies on a ray that emanates from $o_{3}$ and crosses $\widehat{C}_{3}(u x)$. In other words, $o_{4} \in \angle u o_{3} x$. Therefore, $o_{4} \notin \angle p o_{3} o_{1}$, a contradiction.

Assume next that $f \in \widehat{C}_{2}(a u)$. The preceding claim implies that $C_{2} \cap D_{4} \subset \widehat{C_{2}}(a u)$. By Observation 3.5, $o_{4}$ lies on a ray that emanates from $o_{2}$ and crosses $\widehat{C}_{2}(a u)$. Therefore, $o_{4} \notin \angle P o_{2} o_{1}$, again a contradiction.

Case 3: $D_{3} \supset L_{1}$ and $D_{4} \supset L_{1}$. Again, rotate the plane so that the line $\overline{o_{1} O_{2}}$ is horizontal and $o_{2}$ is to the right of $o_{1}$, and assume that $o_{3}$ is in the closed halfplane above $\overline{o_{1} O_{2}}$. We have to show that $o_{4}$ is not inside the convex unbounded region $K$ that is bounded by the rays $\overrightarrow{o_{3} O_{1}}, \overrightarrow{o_{3} O_{2}}$ and the line segment $\left[o_{1} o_{2}\right]$.

Note that in this case $C_{4}$ and $C_{2}$ must intersect. Indeed, we have assumed that $D_{4} \supset$ $D_{2} \backslash$ int $D_{1}$. Thus, if $C_{4}$ does not interset $C_{2}$, then $D_{4} \supset C_{2}$. However, then, since $C_{2} \backslash D_{3} \neq \emptyset, C_{2}$ has to intersect the interior of $L_{2}=D_{4} \backslash$ int $D_{3}$, which is impossible.

Denote by $\gamma$ the $\operatorname{arc} C_{2} \cap D_{4}$. Denote by $p$ the intersection point of $\overrightarrow{o_{3} O_{2}}$ with $C_{2}$ that lies outside $D_{3}$; this is the point on $C_{2}$ farthest from $o_{3}$ (see Fig. 4).

Clearly, $p \notin D_{3}$. Since $L_{2}=D_{4} \backslash$ int $D_{3}$ is a lune, it follows that $p \notin D_{4}$ (for otherwise $C_{2}$ would have to intersect the interior of $L_{2}$ ). We conclude that $p \notin \gamma$. Denote by $q, s$ the intersection points of $\overline{o_{1} O_{2}}$ with $C_{2}$, so that $s$ is outside $D_{1}$; the point $s$ is the midpoint of the $\operatorname{arc} C_{2} \backslash D_{1}$. Since $D_{4} \supset L_{1}$, we have $s \in \gamma$. Denote by $r$ the midpoint of $\gamma$. Since $o_{3}$ is above or on $\overline{o_{1} O_{2}}, p$ is below or on $\overline{o_{1} O_{2}}$. The point $r$ cannot lie on $\widehat{C_{2}}(q p)$, for otherwise, since $p \notin \gamma$, the subarc of $\gamma$ between $r$ and $s$ contains the intersection of $C_{2}$ with the closed halfplane above $\overline{O_{1} O_{2}}$, which is impossible since $r$ is the midpoint of $\gamma$. Hence, $o_{4}$, which lies on $\overrightarrow{o_{2} r}$ (by Observation 3.5), is outside $\angle p o_{2} o_{1}$. This completes the proof.

The planarity of $G$ already implies that $\mathcal{C}$ determines at most $3 n-6$ lunes (unless $\mathcal{C}$ is a nondegenerate pencil, in which case $G$ contains multiple edges; however, in this case $\mathcal{C}$ is easily seen to have exactly $2 n-2$ lunes). We can, however, improve this bound and make it tight $(2 n-2)$, by observing that $G$ is almost a bipartite graph. This is the goal of the remainder of this section. 


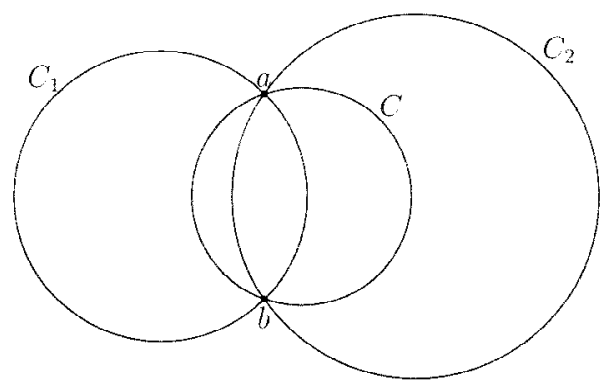

Fig. 5. The configuration in Claim 3.6.

Claim 3.6. Let $C, C_{1}, C_{2}$ be three distinct pairwise-intersecting circles. Suppose that $L_{1}=D_{1} \backslash$ int $D$ and $L_{2}=D \backslash$ int $D_{2}$ are lunes. Then $C_{2}$ passes through the two intersection points of $C$ and $C_{1}$. Moreover, the inner arc of $L_{1}$ is the outer arc of $L_{2}$.

Proof. See Fig. 5. Denote by $a$ and $b$ the intersection points of $C$ and $C_{1}$ (note that $a \neq b$ ). The arc $C_{2} \cap D$ is the inner arc of $L_{2}$, hence $C_{1}$ and $C_{2}$ cannot intersect inside the interior of $D$. The $\operatorname{arc} C_{1} \backslash D$ is the outer $\operatorname{arc}$ of $L_{1}$, hence $C_{1}$ and $C_{2}$ cannot intersect outside $D$. Therefore $C_{1} \cap C_{2} \subset C$, which implies that $C_{2}$ passes through $a$ and $b$.

For the second part, observe that since $C_{2}$ passes through $a$ and $b$, the outer $\operatorname{arc}$ of $L_{2}$ is either $C \cap D_{1}$ (i.e., the inner arc of $L_{1}$ ) or $C \backslash D_{1}$. Assume to the contrary that the outer arc of $L_{2}$ is $C \backslash D_{1}$. Then $D_{2}$ contains $C \cap D_{1}$ which is the inner arc of $L_{1}$. Since $L_{1}$ is a lune, $D_{2}$ must contain also the outer arc of $L_{1}$ which is $C_{1} \backslash D$. Since $D \backslash D_{2}$ is a lune, $D_{2}$ must contain $C_{1} \cap D$ (for otherwise $C_{1}$ intersects the interior of $D \backslash D_{2}$ ). The last two containments imply that $D_{2} \supset D_{1}$ which is impossible since $C_{1}$ and $C_{2}$ intersect at $a$ and $b$.

Lemma 3.7. Suppose that $C \in \mathcal{C}$ supports an inner arc of a lune $L_{1}$, as well as an outer arc of a lune $L_{2}$. Then either $\mathcal{C}$ is a pencil or $C$ supports exactly one inner arc of a lune and one outer arc of a lune. Moreover, if $\mathcal{C}$ is not a pencil, then the inner arc of $L_{1}$ is the outer arc of $L_{2}$.

Proof. Write $L_{1}=D_{1} \backslash$ int $D, L_{2}=D \backslash$ int $D_{2}$, for a (not necessarily distinct) pair of circles $C_{1}, C_{2} \in \mathcal{C}$. Denote by $a$ and $b$ the intersection points of $C$ and $C_{1}$. If $C_{1}=C_{2}$, then $a, b$ are the only intersection points on $C_{1}$ (as well as on $C$ ), because $C_{1} \backslash D$ is the outer arc of $L_{1}$ and $C_{1} \cap D$ is the innner arc of $L_{2}$. Hence $\mathcal{C}$ is a pencil in this case. We may thus assume that $C_{1} \neq C_{2}$.

By Claim 3.6, $C_{2}$ passes through $a, b$ and the inner arc of $L_{1}$ is the outer $\operatorname{arc}$ of $L_{2}$. Denote that arc by $d$. If $C$ contains another inner or outer arc of some lune, then, by the preceding argument, this arc equals $d$. However, $d$ can be an inner arc of at most one lune and an outer arc of at most one lune.

Proof of Theorem 3.1. We prove the theorem by induction on $n$. The theorem clearly holds for $n=2$. Assume that it holds for all $n^{\prime}<n$ and consider the case of $n$ circles. 
Denote by $\mathcal{C}^{+}$the set of all circles in $\mathcal{C}$ that support the outer arc of some lune. Denote by $\mathcal{C}^{-}$the set of all circles in $\mathcal{C}$ that support the inner arc of some lune.

Case 1: $\mathcal{C}^{+} \cap \mathcal{C}^{-}=\emptyset$. In this case $G$ is bipartite. As is well known, bipartite planar graphs on $n$ vertices have at most $2 n-4$ edges, so the theorem holds in this case.

Case 2: $\mathcal{C}^{+} \cap \mathcal{C}^{-} \neq \emptyset$. Let $C$ be a circle in $\mathcal{C}^{+} \cap \mathcal{C}^{-}$. By Lemma 3.7, either $\mathcal{C}$ is a pencil or $C$ supports exactly one inner arc of a lune $L_{1}=D_{1} \backslash$ int $D$ and one outer arc of a lune $L_{2}=D \backslash D_{2}$, and we have $C \cap D_{1}=C \backslash$ int $D_{2}$. If $\mathcal{C}$ is a (nondegenerate) pencil, then clearly it admits exactly $2 n-2$ lunes. If $\mathcal{C}$ is not a pencil, then $\mathcal{C}^{\prime}=\mathcal{C} \backslash\{C\}$ has exactly one lune less than $\mathcal{C}$; indeed, the lunes $L_{1}, L_{2}$ no longer exist, but instead we gained the lune $D_{1} \backslash$ int $D_{2}$. By the induction hypothesis, there are at most $2(n-1)-2$ lunes in $\mathcal{C}^{\prime}$ and therefore at most $2 n-3 \leq 2 n-2$ lunes in $\mathcal{C}$. This establishes the induction step and thus completes the proof.

\subsection{The Number of Lenses in a Family of Pairwise-Intersecting Circles}

In this subsection we prove the following theorem.

Theorem 3.8. A family of $n$ pairwise-intersecting circles in the plane determines at most $18 n$ lenses.

Proof. Let $\mathcal{C}$ be a family of $n$ pairwise-intersecting circles in the plane. We prove the theorem by induction on $n$. The theorem clearly holds for $n \leq 36$, because the number of lenses is at most $\left(\begin{array}{l}n \\ 2\end{array}\right)<18 n$, for $n \leq 36$. Suppose that the theorem holds for all $n^{\prime}<n$ and consider the case of $n>36$ circles.

Lemma 3.9. There exists a point interior to at least $n / 9$ of the disks bounded by circles in $\mathcal{C}$.

Proof. Let $C_{0} \in \mathcal{C}$ be a circle that has the smallest radius $r$. Let $D_{0}^{*}$ be the disk of radius $3 r$ that is concentric with $C_{0}$. For any circle $C \in \mathcal{C} \backslash\left\{C_{0}\right\}$, the area of $D \cap D_{0}^{*}$ is minimized when the radius of $C$ is $r$ and $C$ is fully contained in $D_{0}^{*}$. This minimum area is $\pi r^{2}$. Since the area of $D_{0}^{*}$ is $9 \pi r^{2}$, there is a point inside it that is interior to at least $n / 9$ of the circles in $\mathcal{C}$.

The constant 9 can be improved to 4, as discussed in Theorem 6.1 of [10].

Without loss of generality, assume that the origin, $O$, is interior to at least $n / 9$ of the circles in $\mathcal{C}$.

We perform an inversion $I$ of the plane with respect to $O$, effected by the mapping $I(z)=1 / \bar{z}$, using the complex number representation of the plane. This is a one-toone continuous mapping from the plane (minus the origin) to itself. $I$ maps circles, not passing through the origin, to circles. If $C$ is a circle such that $O \notin D$, then $I$ maps int $D$ onto int $I(C)$. If $C$ is a circle such that $O \in D$, then $I$ maps int $D$ onto the complement of $I(C)$. 
Observation 3.10. Assume that $C_{1}, C_{2} \in \mathcal{C}$, and $O \in$ int $D_{1} \backslash D_{2}$. Let $C_{1}^{\prime}=I\left(C_{1}\right)$ and $C_{2}^{\prime}=I\left(C_{2}\right)$. If $D_{1} \cap D_{2}$ is a lens, then $D_{2}^{\prime} \backslash$ int $D_{1}^{\prime}$ is a lune.

Denote by $\mathcal{C}^{\prime}$ the set of all circles $C \in \mathcal{C}$ such that $O \in D$. We have $\left|\mathcal{C}^{\prime}\right| \geq n / 9$. Since the intersection of all the disks bounded by the circles in $\mathcal{C}^{\prime}$ has a nonempty interior, there is at most one lens that is supported by two circles in $\mathcal{C}^{\prime}$. Denote by $\ell$ the number of lenses supported by a circle in $\mathcal{C}^{\prime}$ and a circle in $\mathcal{C} \backslash \mathcal{C}^{\prime}$. After performing the inversion $I$, we have, by Observation 3.10, at least $\ell$ lunes in the family $I(\mathcal{C})$. By Theorem 3.1, $\ell \leq 2 n-2$.

By the induction hypothesis, the family $\mathcal{C} \backslash \mathcal{C}^{\prime}$ determines at most $18(1-1 / 9) n=16 n$ lenses. Hence, $\mathcal{C}$ determines at most $16 n+(2 n-2)+1<18 n$ lenses. This establishes the induction step and thus completes the proof of the theorem.

In Section 3.4 we shall need the following extension of Theorem 3.8:

Lemma 3.11. Let $A$ and $B$ be two families of circles in the plane, such that every circle in $A$ intersects every circle in $B$, and there is a point $p$ that is interior to all the disks bounding the circles of $A$. Then the number of lenses within the family $A \cup B$ that are supported by a circle of $A$ and by a circle of $B$ is $O(|A|+|B|)$.

Proof. First note that we may assume, without loss of generality, that every pair of circles in $A$ intersect. Indeed, if $C_{1}, C_{2} \in A$ and $C_{1} \cap C_{2}=\emptyset$, then, since $p \in D_{1} \cap D_{2}$, it must be the case that one of $D_{1}, D_{2}$ contains the other disk. Suppose that $D_{1} \subset D_{2}$. We claim that there is no lens that is supported by $C_{2}$ and by a circle in $B$. Indeed, assume that there exists $C \in B$ such that $D \cap D_{2}$ is a lens. Since $C_{1} \subset D_{2}$, we have $C_{1} \cap D \subset D_{2} \cap D$, which means that the $\operatorname{arc} C_{1} \cap D$ is contained within the lens $D_{2} \cap D$, which contradicts the definition of a lens. Therefore, we may exclude $C_{2}$ from $A$ without decreasing the number of lenses under consideration. Hence we may assume that every pair of circles in $A$ intersect.

Perform an inversion map $I$ with respect to $p$. By Observation 3.10, every lens that is supported by a circle in $A$ and a circle in $B$ becomes a lune, unless it contains the point $p$. Moreover, the outer (resp. inner) arc of each such lune is supported by the image of a circle in $B$ (resp. in $A$ ). Clearly, at most one lens can contain $p$.

Denote by $I(A)$ and $I(B)$ the two families that contain the images of the circles of $A$ and of $B$, respectively, under the inversion $I$.

Every pair of circles in $I(A)$ intersect, and each circle of $I(A)$ intersects every circle of $I(B)$. Define a bipartite graph $G$ whose vertices are the circles in $I(A) \cup I(B)$, and whose edges are the pairs $\left(C, C^{\prime}\right)$, where $C \in I(A), C^{\prime} \in I(B)$ and $D^{\prime} \backslash$ int $D$ is a lune within the family $I(A) \cup I(B)$. By Lemma $3.4, G$ is a planar graph. Hence, the number of edges of $G$, which is equal to the number of lunes, the outer arc of which is supported by a circle from $I(B)$ and the inner arc of which is supported by a circle in $I(A)$, is at most $2(|A|+|B|)-4$. Adding the one possible lens that contains $p$, we obtain the asserted bound. 


\subsection{The Number of Lenses in Arrangements of Unit Circles}

We now consider the case of unit circles, and tackle Conjecture 1.1(a).

Our first result shows that the number of lenses in $\mathcal{A}(\mathcal{C})$ is subquadratic. We note that the weaker subquadratic bound $O\left(n^{3 / 2}\right)$ is easy to establish using a forbidden subgraph argument. (An even weaker bound of $O\left(n^{5 / 3}\right)$ follows from the more general results of Tamaki and Tokuyama [20] mentioned in the Introduction.)

Theorem 3.12. The number of lenses in $\mathcal{A}(\mathcal{C})$ is $O\left(n^{4 / 3} \log n\right)$.

Proof. Let $P$ denote the set of centers of the circles in $\mathcal{C}$ and let $\mathcal{D}$ be the set of disks of radius 2 centered at the points of $P$ (each disk in $\mathcal{D}$ is concentric with a circle of $\mathcal{C}$ and its radius is twice as large).

Let $G$ be the bipartite containment subgraph of $\mathcal{D} \times P$; that is, the edges of $G$ are all pairs $(D, p) \in \mathcal{D} \times P$ such that $p \in D$. We apply the batched range-searching technique of Katz and Sharir [12] to $\mathcal{D}$ and $P$. This technique computes $G$ and represents it as the disjoint union of complete bipartite graphs $\left\{\mathcal{D}_{i} \times P_{i}\right\}$, so that $\sum_{i}\left(\left|\mathcal{D}_{i}\right|+\left|P_{i}\right|\right)=$ $O\left(n^{4 / 3} \log n\right)$.

Note that for each lens incident to circles $C, C^{\prime}$, the center $p^{\prime}$ of $C^{\prime}$ lies in the disk $D$ of radius 2 concentric with $C$. Hence $\left(D, p^{\prime}\right)$ appears in one of the graphs $\mathcal{D}_{i} \times P_{i}$.

Hence it suffices to show that the number of lenses "within" each of the graphs $\mathcal{D}_{i} \times P_{i}$ is linear in $\left|\mathcal{D}_{i}\right|+\left|P_{i}\right|$. (Note that a lens $\varphi$ in $\mathcal{A}(\mathcal{C})$ is also a lens in the arrangement of any subset of $\mathcal{C}$ that contains the two circles incident to $\varphi$.) More precisely, let $\mathcal{C}_{i}$ denote the set of circles in $\mathcal{C}$ that are concentric with the disks in $\mathcal{D}_{i}$, and let $\overline{\mathcal{C}}_{i}$ denote the set of circles of $\mathcal{C}$ centered at the points of $P_{i}$. Our goal is to estimate the number of lenses in $\mathcal{C}_{i} \cup \overline{\mathcal{C}}_{i}$

Since every "bichromatic" pair of circles in $\mathcal{C}_{i} \times \overline{\mathcal{C}}_{i}$ intersect, the centers of the circles in $\mathcal{C}_{i} \times \overline{\mathcal{C}}_{i}$ all lie in some square $R$ of side at most 8 . We partition $R$ into 64 small subsquares, each of side 1 , and observe that any pair of circles centered at the same subsquare intersect each other. Now, instead of considering the set $\mathcal{C}_{i} \cup \overline{\mathcal{C}}_{i}$, consider the $O(1)$ sets $\mathcal{C}_{i}^{(p)} \cup \overline{\mathcal{C}}_{i}^{(q)}$, where $\mathcal{C}_{i}^{(p)}$ is the set of circles of $\mathcal{C}_{i}$ whose centers lie in the $p$ th small subsquare, and $\overline{\mathcal{C}}_{i}^{(q)}$ is the set of circles of $\overline{\mathcal{C}}_{i}$ whose centers lie in the $q$ th small subsquare. Since each pair of circles in $\mathcal{C}_{i}^{(p)} \cup \overline{\mathcal{C}}_{i}^{(q)}$ intersect, it follows from Theorem 2.2 that the number of lenses in that set is $O\left(\left|\mathcal{C}_{i}^{(p)}\right|+\left|\overline{\mathcal{C}}_{i}^{(q)}\right|\right)$. Summing these bounds over all $p, q$, we conclude that the number of lenses in $\mathcal{C}_{i} \cup \overline{\mathcal{C}}_{i}$ is $O\left(\left|\mathcal{C}_{i}\right|+\left|\overline{\mathcal{C}}_{i}\right|\right)$. This completes the proof of the theorem.

Remark. We conjecture that the real bound on the number of lenses is near-linear in $n$. However, proving such a bound is likely to be very hard. This is suggested by the following consideration. Let $S$ be a set of $n$ points in the plane, and let $\mathcal{C}$ be the family of unit circles centered at the points of $S$. For a pair of points $p, q \in S$, the distance $|p q|$ is 2 if and only if the two circles centered at $p$ and $q$ are externally tangent to each other. If no two of these points of tangency coincide then, by perturbing the points of $S$ slightly and randomly, we can ensure that at least a constant fraction of the number of these tangencies become lenses in the perturbed arrangement. The best known upper 
bound for the number of repeated distances in a set of $n$ points in the plane is $O\left(n^{4 / 3}\right)$ [17] (see also [13] and [14]), whereas the best known construction gives only a slightly superlinear number of repeated distances [13]. This upper bound has resisted any attempt of improvement for the past 15 years. Hence, improving our bound on the number of lenses below $O\left(n^{4 / 3}\right)$ is likely to be hard. We feel confident, though, that it should not be too difficult to improve the bound to $O\left(n^{4 / 3}\right)$. (We note, though, that, because of the issue of possibly coinciding tangencies, the repeated distances problem is not fully reducible to the lenses problem.)

\subsection{The Number of Lenses and Lunes in Arrangements of Arbitrary Circles}

In this subsection we study general arrangements of circles of arbitrary radii in the plane, and tackle Conjecture 1.1(d). We first have the following upper bound on the number of lenses and lunes in such an arrangement.

Theorem 3.13. The number of lenses and lunes determined by a family of $n$ circles of arbitrary radii in the plane is $O\left(n^{3 / 2+\varepsilon}\right)$, for any $\varepsilon>0$, where the constant of proportionality depends on $\varepsilon$.

Proof. Let $\mathcal{C}$ be a family of $n$ circles of arbitrary radii. Let $G$ be the intersection graph of $\mathcal{C}$. That is, the vertices of $G$ are the circles of $\mathcal{C}$ and the edges of $G$ connect all intersecting pairs of circles.

We run a batched range-searching procedure for constructing $G$ and for representing it as the disjoint union of a family of complete bipartite graphs $\left\{\mathcal{A}_{i} \times \mathcal{B}_{i}\right\}$. A standard way of doing this is as follows. Represent a circle $C$ whose center is at $(a, b)$ and whose radius is $r$ by the point

$$
p_{C}\left(a, b, r,-\left(r^{2}-a^{2}-b^{2}\right)\right) \in \mathbb{R}^{4},
$$

and by the pair of hyperplanes

$$
\begin{array}{ll}
h_{C}^{+}: & x_{4}=2 a x_{1}+2 b x_{2}+2 r x_{3}+\left(r^{2}-a^{2}-b^{2}\right), \\
h_{C}^{-}: & x_{4}=2 a x_{1}+2 b x_{2}-2 r x_{3}+\left(r^{2}-a^{2}-b^{2}\right) .
\end{array}
$$

Note that a circle $C$ of radius $r$ centered at $(a, b)$ and a circle $C^{\prime}$ of radius $R$ centered at $(\xi, \eta)$ intersect if and only if

$$
(R-r)^{2} \leq(a-\xi)^{2}+(b-\eta)^{2} \leq(R+r)^{2},
$$

or

$$
2 a \xi+2 b \eta+2 r R+\left(r^{2}-a^{2}-b^{2}\right) \geq-\left(R^{2}-\xi^{2}-\eta^{2}\right)
$$

and

$$
2 a \xi+2 b \eta-2 r R+\left(r^{2}-a^{2}-b^{2}\right) \leq-\left(R^{2}-\xi^{2}-\eta^{2}\right) .
$$

In other words, they intersect if and only if the point $p_{C}$ lies above $h_{C^{\prime}}^{-}$and below $h_{C^{\prime}}^{+}$. 
Hence, the range-searching problem that we face is: We have a set $P$ of $n$ points in $\mathbb{R}^{4}$, all lying on the paraboloid $\pi: x_{4}=x_{1}^{2}+x_{2}^{2}-x_{3}^{2}$, and a set $W$ of $n$ wedges, we wish to find a compact representation of the set of all pairs of point-wedge containment. Applying standard range-searching machinery (see, e.g., [1] and [2]), we can represent the set of these pairs as the disjoint union of a family of complete bipartite graphs $\left\{P_{i} \times W_{i}\right\}$, such that the overall size of the vertex sets of these graphs is $O\left(n^{3 / 2+\varepsilon}\right)$, for any $\varepsilon>0$, with the constant of proportionality depending on $\varepsilon$. We then transform each of the graphs $P_{i} \times W_{i}$ to the corresponding graph $\mathcal{A}_{i} \times \mathcal{B}_{i}$, where $\mathcal{A}_{i}$ is the set of circles whose representing points are in $P_{i}$ and $\mathcal{B}_{i}$ is the set of circles whose representing wedges are in $W_{i}$.

Clearly, if two of the given circles $C, C^{\prime}$ form a lens or a lune, then they intersect, so the pair $\left(C, C^{\prime}\right)$ appears in one of the bipartite graphs $\mathcal{A}_{i} \times \mathcal{B}_{i}$, and forms a lens or a lune in $\mathcal{A}_{i} \cup \mathcal{B}_{i}$.

We fix a graph $\mathcal{A}_{i} \times \mathcal{B}_{i}$, and denote it as $\mathcal{A} \times \mathcal{B}$ for short. Note that each circle in $\mathcal{A}$ intersects every circle in $\mathcal{B}$, but there may be disjoint pairs of circles in $\mathcal{A} \times \mathcal{A}$ and in $\mathcal{B} \times \mathcal{B}$.

Suppose that the smallest circle in $\mathcal{A} \cup \mathcal{B}$ is $C \in \mathcal{A}$, and let $r$ be the radius of $C$. We argue as in the proof of Lemma 3.9. That is, let $D_{0}$ be the disk of radius $3 r$ concentric with $C$. Each circle $C^{\prime} \in \mathcal{B}$ intersects $C$ and has radius $r^{\prime} \geq r$, so, arguing as above, the intersection of $D_{0}$ with the disk $D^{\prime}$ that $C^{\prime}$ bounds has area at least $\pi r^{2}$. Hence, we can place $O(1)$ points in $D_{0}$ so that any such $D^{\prime}$ contains at least one of them. This implies that we can decompose $\mathcal{B}$ into $O(1)$ families $\mathcal{B}^{(1)}, \ldots, \mathcal{B}^{(p)}$ so that all the circles in the same family have a common point in their interiors.

Lemma 3.11 implies that the number of "bichromatic" lenses in $\mathcal{A} \cup \mathcal{B}^{(j)}$ is $O(|\mathcal{A}|+$ $\left.\left|\mathcal{B}^{(j)}\right|\right)$. The analysis of lunes is a bit more involved. First, as implied by Lemma 3.4, the number of bichromatic lunes whose inner arc is supported by a circle of $\mathcal{B}^{(j)}$ and whose outer arc is supported by a circle of $\mathcal{A}$ is $O\left(|\mathcal{A}|+\left|\mathcal{B}^{(j)}\right|\right)$. (Note that, as in the proof of Lemma 3.11, we first argue that we may assume that every pair of circles in $\mathcal{B}^{(j)}$ intersect; indeed, if this family contains two circles $C, C^{\prime}$ such that $C$ is fully contained in the interior of $C^{\prime}$, then, as is easily verified, $C$ cannot support the inner arc of any lune under consideration, so it can be ignored.)

It remains to consider lunes whose inner arc is supported by a circle $C \in \mathcal{A}$ and whose outer arc is supported by a circle $C^{\prime} \in \mathcal{B}^{(j)}$. Suppose first that the radius of $C$ is smaller than or equal to the radius of $C^{\prime}$. Then the outer arc of the lune is larger than half of $C^{\prime}$, and consequently the number of these lunes is at most $O\left(\left|\mathcal{B}^{(j)}\right|\right)$. Any other lune under consideration has its inner arc supported by a circle in $\mathcal{A}$ whose radius is at least $r$. Let $\mathcal{A}^{\prime}$ denote the subset of these circles. Arguing as above, we can partition $\mathcal{A}^{\prime}$ into $O(1)$ subfamilies, so that all circles in the same subfamily have a common point in their interiors. For each such subfamily $\mathcal{A}^{\prime \prime}$, Lemma 3.4 and the analysis given in the preceding paragraph, imply that the number of bichromatic lunes under consideration in $\mathcal{A}^{\prime \prime} \cup \mathcal{B}^{(j)}$ is $O\left(\left|\mathcal{A}^{\prime \prime}\right|+\left|\mathcal{B}^{(j)}\right|\right)$. Summing this over all the subfamilies $\mathcal{A}^{\prime \prime}$, we finally obtain that the overall number of lenses and lunes in $\mathcal{A} \cup \mathcal{B}^{(j)}$ is $O\left(|\mathcal{A}|+\left|\mathcal{B}^{(j)}\right|\right)$.

Summing this bound over the $O(1)$ indices $j$, we conclude that the number of bichromatic lenses and lunes in $\mathcal{A} \cup \mathcal{B}=\mathcal{A}_{i} \cup \mathcal{B}_{i}$ is $O\left(\left|\mathcal{A}_{i}\right|+\left|\mathcal{B}_{i}\right|\right)$. Summing this bound over all bipartite graphs $\mathcal{A}_{i} \times \mathcal{B}_{i}$ in our decomposition, we conclude that the overall number of lenses and lunes in $\mathcal{C}$ is $O\left(n^{3 / 2+\varepsilon}\right)$, as asserted. 
We next derive the following strengthening of Theorem 3.13:

Theorem 3.14. The number of lenses and lunes determined by a family of $n$ circles of arbitrary radii in the plane with $P$ intersecting pairs is $O\left(n^{1 / 2-\varepsilon} P^{1 / 2+\varepsilon}+n\right)$, for any $\varepsilon>0$, where the constant of proportionality depends on $\varepsilon$.

Proof. Clearly, we only need to prove the theorem in the case that $P=o\left(n^{2}\right)$, and we may also assume that $P>n$, for otherwise the complexity of the arrangement is $O(n)$, so the theorem trivially holds in this case. Put $r=n^{2} / P$, and choose a random sample $\mathcal{R}$ of $r$ circles of $\mathcal{C}$. The expected number of intersecting pairs in $\mathcal{R}$ is $O\left(\mathrm{Pr}^{2} / n^{2}\right)=O(r)$, which implies that the expected complexity of $\mathcal{A}(\mathcal{R})$ is $O(r)$. Decompose $\mathcal{A}(\mathcal{R})$ into pseudo-trapezoids (as in [16]). The $\varepsilon$-net theory implies that, with high probability, each pseudo-trapezoid is crossed by at most $O((n / r) \log r)$ circles of $\mathcal{C}$. We can thus assume that our sample $\mathcal{R}$ is such that the number of pseudo-trapezoids is $O(r)$ and each is crossed by at most $O((n / r) \log r)$ circles of $\mathcal{C}$. For any lens or lune $L$ in $\mathcal{A}(\mathcal{C})$ there exists a pseudo-trapezoid $\tau$ such that $L$ is also a lens or lune in $\mathcal{A}\left(\mathcal{C}_{\tau}\right)$, where $\mathcal{C}_{\tau}$ is the collection of circles of $\mathcal{C}$ that cross $\tau$. By Theorem 3.13, the number of lenses and lunes in $\mathcal{A}\left(\mathcal{C}_{\tau}\right)$ is $O\left((n / r)^{3 / 2+\varepsilon}\right)$, for any $\varepsilon>0$. Hence, the total number of lenses and lunes in $\mathcal{A}(\mathcal{C})$ is

$$
O(r) \cdot O\left((n / r)^{3 / 2+\varepsilon}\right)=O\left(n^{3 / 2+\varepsilon} / r^{1 / 2+\varepsilon}\right)=O\left(n^{1 / 2-\varepsilon} P^{1 / 2+\varepsilon}\right),
$$

as asserted.

Remark. We do not know whether the bound in Theorem 3.13 is tight. The best lower bound that we can construct is $\Omega\left(n^{4 / 3}\right)$. Indeed, construct a set $\mathcal{L}$ of $n$ lines and a set $P$ of $n$ points that have $\Theta\left(n^{4 / 3}\right)$ incidences between them (see, e.g., [11] for such a construction). Choose a sufficiently small parameter $\delta>0$, replace each point $p \in P$ by a circle of radius $\delta$ centered at $p$, and replace each line $\ell \in \mathcal{L}$ by a parallel line that lies above $\ell$ at distance $\delta$ from it. We now have $\Theta\left(n^{4 / 3}\right)$ tangencies between the new circles and lines. Finally, take each of the new lines, move it slightly down and bend it slightly upwards into a huge circle. It is easily seen that these deformations can be made so that all the huge circles have the same radius and so that each of the former tangencies is turned into a lens in the new arrangement. We thus obtain an arrangement of $2 n$ circles, of only two different radii, that has $\Omega\left(n^{4 / 3}\right)$ lenses. (Similarly, by bending the lines slightly downwards, we can obtain an arrangement with $\Omega\left(n^{4 / 3}\right)$ lunes.)

\section{The Existence of Vertices Incident to at Most Three Circles}

In this section we tackle parts (a), (c) and (d) of Conjecture 1.1, and derive partial solutions to them.

\subsection{Vertices of Low Degree for Pairwise-Intersecting Circles}

In this subsection we establish the following result: 
Theorem 4.1. LetC be a family of $n$ pairwise-intersecting circles in the plane. If $n$ is sufficiently large and $\mathcal{C}$ is not a pencil, then there exists an intersection point incident to at most three circles.

We need the following easy consequence of Euler's formula for planar maps, which has already been used in the previous paper [15]; we include the simple proof for the sake of completeness.

Lemma 4.2. Let $\mathcal{C}$ be a finite family of circles in the plane. For every $k \geq 2$ denote by $t_{k}$ the number of vertices of $\mathcal{A}(\mathcal{C})$ that are incident to exactly $k$ circles of $\mathcal{C}$. Denote by $f_{k}(k \geq 1)$ the number of faces of $\mathcal{A}(\mathcal{C})$ that have $k$ edges. Then

$$
t_{2}+f_{2}+2 f_{1} \geq 6+\sum_{k \geq 3}(k-3) t_{k}+\sum_{k \geq 3}(k-3) f_{k} .
$$

Proof. Denote by $V, E, F$ the numbers of vertices, edges and faces of $\mathcal{A}(\mathcal{C})$, respectively. We have

$$
V=\sum_{k \geq 2} t_{k}, \quad F=\sum_{k \geq 1} f_{k}, \quad E=\sum_{k \geq 2} k t_{k}=\frac{1}{2} \sum_{k \geq 1} k f_{k} .
$$

By Euler's formula, $V+F=E+1+c$, where $c$ is the number of connected components of $\cup \mathcal{C}$. Therefore,

$$
3 \sum_{k \geq 2} t_{k}+3 \sum_{k \geq 1} f_{k}=\sum_{k \geq 2} k t_{k}+\sum_{k \geq 1} k f_{k}+3+3 c,
$$

which is easily seen to imply the lemma.

Proof of Theorem 4.1. Let $t_{k}, f_{k}$, for $k \geq 2$, be as defined in Lemma 4.2. Note that we may assume in this case that $f_{1}=0$. Indeed, if there is a circle which contains just one intersection point, then it follows from the fact that the circles in $\mathcal{C}$ are pairwise intersecting, that $\mathcal{C}$ is a degenerate pencil, contrary to assumption.

We assume to the contrary that $t_{2}=t_{3}=0$ and derive a contradiction. Under this assumption, Lemma 4.2 implies

$$
V=\sum_{k \geq 4} t_{k} \leq \sum_{k \geq 4}(k-3) t_{k} \leq f_{2}-6 .
$$

By Theorems 3.1 and 3.8, the number of bounded faces of $\mathcal{A}(\mathcal{C})$ of degree 2 (i.e., the lunes and lenses of $\mathcal{C}$ ) is less than 20 n. Taking into account the unbounded face as well, we still have $V \leq f_{2}-6<20 n$.

Claim 4.3. $\quad \mathcal{C}$ does not contain a pencil of size $\geq 9 n^{1 / 2}$.

Proof. Suppose to the contrary that there exists a pencil $\mathcal{C}^{\prime} \subset \mathcal{C}$ of size $\left|\mathcal{C}^{\prime}\right|=k \geq 9 n^{1 / 2}$. Each circle in $\mathcal{C} \backslash \mathcal{C}^{\prime}$ intersects the circles in $\mathcal{C}^{\prime}$ in at least $k$ distinct points. Hence, if we 
add a circle $C_{1}$ of $\mathcal{C} \backslash \mathcal{C}^{\prime}$ to $\mathcal{C}^{\prime}$ we obtain at least $k$ new intersection points. Adding another circle $C_{2} \in \mathcal{C} \backslash \mathcal{C}^{\prime}$ yields at least $k-2$ additional new intersection points with the circles in $\mathcal{C}^{\prime}$ (note that $C_{1}$ and $C_{2}$ can share at most two of these intersection points). Continuing in this manner, adding the $j$ th circle of $\mathcal{C} \backslash \mathcal{C}^{\prime}$ will yield at least $k-2 j+2$ new intersection points.

Suppose first that $k<2 n / 3$. Then we can add $k / 2$ circles of $\mathcal{C} \backslash \mathcal{C}^{\prime}$ to $\mathcal{C}^{\prime}$, and obtain at least $k^{2} / 4$ distinct vertices of $\mathcal{A}(\mathcal{C})$. Since the number of vertices is at most $20 n$, we obtain $k<9 n^{1 / 2}$, a contradiction.

Suppose then that $k \geq 2 n / 3$. Adding one circle $C \in \mathcal{C} \backslash \mathcal{C}^{\prime}$ to $\mathcal{C}^{\prime}$ yields at least $2 n / 3$ new intersection points, all having degree 2 in $\mathcal{A}\left(\mathcal{C}^{\prime} \cup\{C\}\right)$. Since each of these points must have degree at least 4 in $\mathcal{A}(\mathcal{C})$, it follows that $\mathcal{C} \backslash \mathcal{C}^{\prime}$ must contain at least $2 n / 3$ additional circles, a contradiction that completes the proof of the claim.

Since $f_{2} \leq 20 n$ it follows that by removing at most $20 n$ edges from $\mathcal{A}(\mathcal{C})$ we obtain a planar graph without multiple edges. Since the number of edges in such a planar graph is at most three times the number of its vertices, we obtain $E-20 n<3 V$, or $E<80 n$.

Claim 4.4. If $n$ is sufficiently large, then each vertex of $\mathcal{A}(\mathcal{C})$ is incident to at most $27 n^{3 / 4}$ circles.

Proof. Suppose to the contrary that there exists an intersection point $p$ incident to more than $27 n^{3 / 4}$ circles. Let $\mathcal{C}^{\prime}$ denote the subfamily of circles incident to $p$.

By Claim 4.3, $\mathcal{C}$ does not contain a pencil of size $9 n^{1 / 2}$. Therefore, within the family $\mathcal{C}^{\prime}$, every intersection point other than $p$ has degree at most $9 n^{1 / 2}$. Hence each circle $C \in \mathcal{C}^{\prime}$ is incident to at least $\left(27 n^{3 / 4}\right) /\left(9 n^{1 / 2}\right)=3 n^{1 / 4}$ distinct intersection points, so $C$ contributes at least these many edges to $\mathcal{A}(\mathcal{C})$. Hence, the number of edges of $\mathcal{A}(\mathcal{C})$ is at least $27 n^{3 / 4} \cdot 3 n^{1 / 4}=81 n$, a contradiction.

By Claim 4.4, each circle in $\mathcal{C}$ is incident to at least $n /\left(27 n^{3 / 4}\right)>\frac{1}{27} n^{1 / 4}$ distinct intersection points, and thus contributes at least these many edges to $\mathcal{A}(\mathcal{C})$. Hence the number of edges of $\mathcal{A}(\mathcal{C})$ is at least $\frac{1}{27} n^{5 / 4}$, which is greater than $80 n$ when $n$ is sufficiently large. This contradiction completes the proof of the theorem.

Theorem 4.1 is a partial solution to Conjecture 1.1(d). One may say that Conjecture 1.1(d) is tight, in the sense that we cannot guarantee the existence of vertices incident to only two circles. We have already seen in [15] that there are configurations of four pairwise-intersecting unit circles such that every intersection point is incident to exactly three circles. If we do not restrict ourselves to unit circles, we also have the example in Fig. 6 of six circles where each intersection point is incident to at least three circles. This example also has the property that at most two circles pass through any two distinct points (i.e., no pencils of size $>2$ exist). We can add a seventh circle (the dotted one in Fig. 6) that violates this condition but preserves the property that each intersection point is incident to at least three circles. 


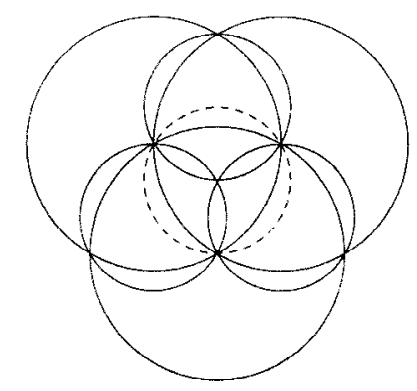

Fig. 6. An arrangement of six or seven circles where each vertex is incident to at least three circles.

\subsection{Vertices of Low Degree in Arrangements of Unit Circles}

In this subsection we establish the following result, which provides a partial solution to Conjecture 1.1(a) posed in the Introduction.

Theorem 4.5. Let $\mathcal{C}$ be a collection of $n$ unit circles in the plane. If the number of pairs of intersecting circles in $\mathcal{C}$ is at least $\beta n$, for some sufficiently large constant $\beta$, then $\mathcal{A}(\mathcal{C})$ contains a vertex incident to at most three circles.

Proof. We assume to the contrary that $\mathcal{A}(\mathcal{C})$ does not contain any such vertex, and derive a linear upper bound on $P$, the number of intersecting pairs of circles. There exists a circle $C \in \mathcal{C}$ that intersects at least $2 P / n$ other circles of $\mathcal{C}$. Let $\sigma_{0}$ denote a unit disk that intersects the maximum number, $\xi$, of circles of $\mathcal{C}$; clearly, $\xi \geq 2 P / n$, or $P \leq \xi n / 2$. Denote the set of these circles by $\mathcal{C}_{\sigma_{0}}$. The centers of all circles of $\mathcal{C}_{\sigma_{0}}$ lie in the disk $\sigma_{0}^{*}$ that is concentric with $\sigma_{0}$ and has radius 2 (note that any circle centered in $\sigma_{0}^{*}$ belongs to $\mathcal{C}_{\sigma_{0}}$ ). Cover $\sigma_{0}^{*}$ by seven unit disks (this is easy to do, using a construction based on the hexagonal grid; see Fig. 7). One of these disks, call it $\sigma_{1}$, contains at least $\xi / 7$ centers. The set $\mathcal{C}_{1}$ of circles centered in $\sigma_{1}$ has the property that every pair of its elements intersect each other, and the intersection points of any such pair lie in the disk $\sigma_{1}^{*}$ of radius 2 concentric with $\sigma_{1}$; the number $P_{1}$ of these pairs is thus at least $\left(\begin{array}{c}\xi / 7 \\ 2\end{array}\right)$. The size $n_{1}$ of $\mathcal{C}_{1}$ satisfies $n_{1} \leq \xi$, as follows from the maximality of $\xi$.

As the subsequent analysis will show, a technical problem may arise when these pairs of circles intersect in too few points, or, more precisely, when there are intersection points of very high degree (linear in $\xi$ ). The following lemma takes care of this problem.

Lemma 4.6. If $\sigma_{1}^{*}$ contains a vertex incident to more than a $\xi$ circles of $\mathcal{C}_{1}$, for any constant parameter $a$, then the number of distinct vertices of $\mathcal{A}(\mathcal{C})$ within $\sigma_{1}^{*}$ is at least $a \xi(a \xi-2) / 2$.

Proof. Let $v$ be a point in $\sigma_{1}^{*}$ incident to $w \geq a \xi$ circles of $\mathcal{C}_{1}$. There may be at most $w / 2$ tangent pairs of these circles, and the other pairs of them intersect at pairwise distinct 


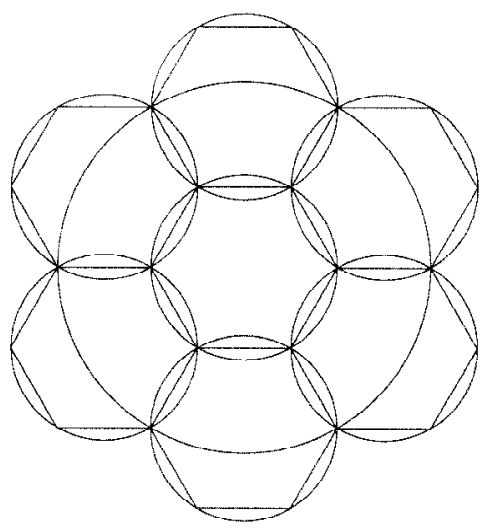

Fig. 7. Covering a disk of radius 2 by seven disks of radius 1 .

points that all lie in $\sigma_{1}^{*}$. The number of these points is thus at least

$$
\left(\begin{array}{l}
w \\
2
\end{array}\right)-\frac{w}{2}=\frac{w(w-2)}{2} \geq \frac{a \xi(a \xi-2)}{2} .
$$

We now cover $\sigma_{1}^{*}$, as above, by seven unit disks. One of them, call it $\sigma$, has the following property:

(i) If the condition of Lemma 4.6 holds, then $\sigma$ contains at least $a \xi(a \xi-2) / 14$ distinct vertices of $\mathcal{A}(\mathcal{C})$.

(ii) Otherwise, at least $\xi(\xi-7) / 686$ pairs of circles of $\mathcal{C}_{1}$ intersect inside $\sigma$.

Let $\mathcal{C}_{\sigma}$ denote the set of circles that intersect $\sigma$. By the maximality property of $\sigma_{0}$, we have $n_{\sigma}=\left|\mathcal{C}_{\sigma}\right| \leq \xi$.

We modify the analysis based on Euler's formula, given in Lemma 4.2, and apply it to the arrangement $\tilde{\mathcal{A}}\left(\mathcal{C}_{\sigma}\right)$, which is obtained by clipping $\mathcal{A}\left(\mathcal{C}_{\sigma}\right)$ to within $\sigma$. Specifically, let $V, E$ and $F$ be the sets of vertices, edges and faces of $\tilde{\mathcal{A}}\left(\mathcal{C}_{\sigma}\right)$. (Note that the intersection points of the circles of $\mathcal{C}_{\sigma}$ with $\partial \sigma$ constitute additional vertices of $\tilde{\mathcal{A}}\left(\mathcal{C}_{\sigma}\right)$. By shifting $\sigma$ slightly, if necessary, we may assume that the number of these new vertices is exactly $2 n_{\sigma}$ and each is incident to exactly one edge of the clipped arrangement.) We have $|V|+|F|=|E|+1+c$, where $c$ is the number of connected components of $\sigma \cap \bigcup \mathcal{C}_{\sigma}$. We also have

$$
|V|=2 n_{\sigma}+\sum_{k \geq 2} t_{k}^{(\sigma)}, \quad|F|=\sum_{k \geq 1} f_{k}^{(\sigma)}, \quad|E|=n_{\sigma}+\sum_{k \geq 2} k t_{k}^{(\sigma)}=\frac{1}{2} \sum_{k \geq 1} k f_{k}^{(\sigma)},
$$

where $t_{k}^{(\sigma)}$ is the number of vertices of $\tilde{\mathcal{A}}\left(\mathcal{C}_{\sigma}\right)$ that lie in the interior of $\sigma$ and are incident to exactly $k$ circles of $\mathcal{C}_{\sigma}$, and $f_{k}^{(\sigma)}$ is the number of faces of $\tilde{\mathcal{A}}\left(\mathcal{C}_{\sigma}\right)$ that are incident to exactly $k$ edges of $\tilde{\mathcal{A}}\left(\mathcal{C}_{\sigma}\right)$, where each edge that terminates on $\partial \sigma$ is counted twice (all these edges bound the unbounded face of the clipped arrangement). Hence we have

$$
6 n_{\sigma}+3 \sum_{k \geq 2} t_{k}^{(\sigma)}+3 \sum_{k \geq 1} f_{k}^{(\sigma)}=n_{\sigma}+\sum_{k \geq 2} k t_{k}^{(\sigma)}+\sum_{k \geq 1} k f_{k}^{(\sigma)}+3+3 c .
$$


Equivalently,

$$
t_{2}^{(\sigma)}+f_{2}^{(\sigma)}+5 n_{\sigma}=\sum_{k \geq 4}(k-3) t_{k}^{(\sigma)}+\sum_{k \geq 4}(k-3) f_{k}^{(\sigma)}+3+3 c .
$$

Since we have assumed that $\mathcal{A}(\mathcal{C})$ does not contain any vertex of degree 2 or 3 , it follows that $t_{2}^{(\sigma)}=t_{3}^{(\sigma)}=0$. We next apply Theorem 3.12 to $\mathcal{C}_{\sigma}$ and observe that the clipping of the arrangement does not affect the asymptotic bound on $f_{2}^{(\sigma)}$ provided by the theorem. Using also the trivial bound $f_{1}^{(\sigma)} \leq n_{\sigma}$, we thus obtain

$$
\sum_{k \geq 4} k t_{k}^{(\sigma)}=O\left(n_{\sigma}^{4 / 3} \log n_{\sigma}\right)=O\left(\xi^{4 / 3} \log \xi\right) .
$$

Suppose first that, in the construction of $\sigma$, the condition of Lemma 4.6 did hold, with a value of $a$ that will be determined later. In this case, as follows from the lemma and from the construction, there are at least $a \xi(a \xi-2) / 14$ distinct vertices of $\mathcal{A}(\mathcal{C})$ inside $\sigma$. In this case (2) implies that

$$
\frac{a \xi(a \xi-2)}{14}<\sum_{k \geq 4} k t_{k}^{(\sigma)}=O\left(\xi^{4 / 3} \log \xi\right)
$$

In other words, $\xi$ is bounded by a constant $c_{1}$ (that depends on $a$ ), so we have $P \leq c_{1} n / 2$.

Suppose then that the condition of Lemma 4.6 did not hold for $a$. That is, no point is incident to more than $a \xi$ circles of $\mathcal{C}_{1}$. We then have

$$
P_{\sigma} \leq \sum_{k=2}^{a \xi}\left(\begin{array}{l}
k \\
2
\end{array}\right) s_{k}^{(\sigma)},
$$

where $P_{\sigma}$ is the number of pairs of circles in $\mathcal{C}_{1}$ that intersect inside $\sigma$, and $s_{k}^{(\sigma)}$ is the number of points that lie inside $\sigma$ and are incident to exactly $k$ circles of $\mathcal{C}_{1}$.

Let $s_{\geq k}^{(\sigma)}$ denote the number of vertices of $\mathcal{A}\left(\mathcal{C}_{1}\right)$ that lie inside $\sigma$ and whose degree is at least $k$, for $k \geq 2$. By the result of Spencer et al. [17] (see also [8] and [18]), one has (recall that $n_{1}=\left|\mathcal{C}_{1}\right|$ )

$$
s_{\geq k}^{(\sigma)} \leq b\left(\frac{n_{1}}{k}+\frac{n_{1}^{2}}{k^{3}}\right),
$$

for an appropriate absolute constant $b$. (See Lemma 4.10 below for a strengthening of this bound, which is not needed for the present analysis.)

Put

$$
P^{*}=\sum_{k=A}^{a \xi}\left(\begin{array}{l}
k \\
2
\end{array}\right) s_{k}^{(\sigma)},
$$

for a constant parameter $A$ that will be determined shortly. We have

$$
P^{*}=\sum_{k=A}^{a \xi}\left(\begin{array}{l}
k \\
2
\end{array}\right)\left[s_{\geq k}^{(\sigma)}-s_{\geq k+1}^{(\sigma)}\right]
$$




$$
\begin{aligned}
& \leq\left(\begin{array}{l}
A \\
2
\end{array}\right) s_{\geq A}^{(\sigma)}+\sum_{k=A+1}^{a \xi}\left[\left(\begin{array}{l}
k \\
2
\end{array}\right)-\left(\begin{array}{c}
k-1 \\
2
\end{array}\right)\right] s_{\geq k}^{(\sigma)} \\
& \leq\left(\begin{array}{l}
A \\
2
\end{array}\right) s_{\geq A}^{(\sigma)}+\sum_{k=A+1}^{\left\lfloor\sqrt{n_{1}}\right\rfloor} k s_{\geq k}^{(\sigma)}+\sum_{k=\left\lfloor\sqrt{n_{1}}\right\rfloor+1}^{a \xi} k s_{\geq k}^{(\sigma)} .
\end{aligned}
$$

Using (4), we readily obtain that

$$
P^{*} \leq \frac{3 b n_{1}^{2}}{A}+2 a b \xi n_{1} \leq\left(\frac{3 b}{A}+2 a b\right) \xi^{2} .
$$

Since $P_{\sigma} \geq \xi(\xi-7) / 686$, it follows that if we choose $A$ sufficiently large and $a$ sufficiently small, we can ensure that $P^{*}<P_{\sigma} / 2$. Using (2), this implies that

$$
\frac{\xi(\xi-7)}{686} \leq P_{\sigma} \leq 2 \sum_{k=2}^{A-1}\left(\begin{array}{l}
k \\
2
\end{array}\right) s_{k}^{(\sigma)} \leq A \sum_{k \geq 2} k s_{k}^{(\sigma)} \leq A \sum_{k \geq 4} k t_{k}^{(\sigma)} \leq B \xi^{4 / 3} \log \xi
$$

for an appropriate constant $B$. (The fourth inequality follows from the observation that any vertex that contributes to the $\operatorname{sum} \sum_{k \geq 2} k s_{k}^{(\sigma)}$ also contributes to the sum $\sum_{k \geq 4} k t_{k}^{(\sigma)}$, with a larger or equal coefficient $k$.) Hence, as above, $\xi$ is at most some constant $c_{2}$, so $P \leq c_{2} n / 2$ in this case. Hence, choosing $\beta>\max \left\{c_{1}, c_{2}\right\} / 2$ we obtain a contradiction, which therefore completes the proof of the theorem.

Inspecting the proof of the theorem, we actually have the following stronger result.

Corollary 4.7. Let $\mathcal{C}$ be a finite family of unit circles with the property that there exists a unit disk that intersects at least $\beta$ circles of $\mathcal{C}$. Then there exists a vertex of $\mathcal{A}(\mathcal{C})$ that is incident to at most three circles.

\subsection{Vertices of Low Degree in Arrangements of Arbitrary Circles}

In this section we establish the following theorem, whose proof exploits the bound on the number of lunes and lenses given in Theorem 3.14.

Theorem 4.8. There exists an absolute constant $\beta$ with the following property. Let $\mathcal{C}$ be a family of $n$ circles of arbitrary radii in the plane, and let $q \geq 2$ denote the maximal size of a pencil in $\mathcal{C}$. If the number of pairs of intersecting circles in $\mathcal{C}$ is at least $\beta n\left(q+n^{1 / 3}\right)$, then $\mathcal{A}(\mathcal{C})$ contains a vertex incident to at most three circles.

Proof. Applying Lemma 4.2 to $\mathcal{A}(\mathcal{C})$, and continuing to use the same notation, we obtain

$$
t_{2}+f_{2}+2 f_{1} \geq 6+\sum_{k \geq 3}(k-3) t_{k}+\sum_{k \geq 3}(k-3) f_{k} .
$$

Note that, as above, $f_{1} \leq n$. Assume to the contrary that $t_{2}=t_{3}=0$. Then we have 
(replacing $\varepsilon$ by $3 \varepsilon$ in Theorem 3.13) $V \leq E \leq 4\left(f_{2}+2 f_{1}\right)=O\left(n^{3 / 2+3 \varepsilon}\right)$, where $V$ and $E$ denote, respectively, the number of vertices and edges of $\mathcal{A}(\mathcal{C})$.

Let $P$ denote the number of pairs of intersecting circles in $\mathcal{C}$. We have

$$
P \leq \sum_{k \geq 4}\left(\begin{array}{l}
k \\
2
\end{array}\right) t_{k}
$$

Denote by $t_{\geq k}$ the number of vertices of $\mathcal{A}(\mathcal{C})$ incident to at least $k$ circles. We need the following result, which improves a bound due to Clarkson et al. [8] on the number of vertices of large degree in arrangements of circles of arbitrary radii.

Lemma 4.9. Let $\mathcal{C}$ be a family of $n$ circles of arbitrary radii in the plane with $P$ pairs of intersecting circles. Then the number $t_{\geq k}$ of points incident to at least $k$ circles satisfies

$$
t_{\geq k} \leq b\left(\frac{P}{k^{2.5}}+\frac{n}{k}\right)
$$

for an appropriate absolute constant $b$.

Proof. The approach is to derive a refined bound on the number $I$ of incidences between the circles of $\mathcal{C}$ and the points in an $m$-element set $M$. This is done using the following variant of the technique of [8].

Draw a random sample $\mathcal{R}$ of $r=\left\lceil n^{2} / P\right\rceil$ circles from $\mathcal{C}$. The expected number of intersecting pairs in $\mathcal{R}$ is at most $P(r / n)^{2}=r$. Decompose $\mathcal{A}(\mathcal{R})$ into pseudo-trapezoids (see [16] for details), and for each pseudo-trapezoid $\tau$ consider the set $M_{\tau}$ of points of $M$ that lie in $\tau_{0}$, which is $\tau$ with its four vertices removed, and the set $\mathcal{C}_{\tau}$ of circles that intersect $\tau_{0}$. Put $m_{\tau}=\left|M_{\tau}\right|$ and $n_{\tau}=\left|\mathcal{C}_{\tau}\right|$. By the results of [8], the number of incidences between $\mathcal{C}_{\tau}$ and $M_{\tau}$ is $O\left(m_{\tau}^{3 / 5} n_{\tau}^{4 / 5}+m_{\tau}+n_{\tau}\right)$. We sum this over all $\tau$ 's, and note that the incidences that we miss are between the circles of $\mathcal{C}$ and the vertices of the trapezoids. Any such incidence can be charged to an intersection between a circle of $\mathcal{R}$ and a circle of $\mathcal{C}$. The expected number of these intersections is $O(P r / n)=O(n)$. Denoting by $I^{\prime}$ the number of these incidences, we obtain

$$
I=I^{\prime}+O\left(\sum_{\tau} m_{\tau}^{3 / 5} n_{\tau}^{4 / 5}+m_{\tau}+n_{\tau}\right) .
$$

Using Hölder's inequality, and observing that $\sum_{\tau} m_{\tau}=O(m)$, we obtain

$$
\begin{aligned}
I & =I^{\prime}+O\left(\left(\sum_{\tau} m_{\tau}\right)^{3 / 5} \cdot\left(\sum_{\tau} n_{\tau}^{2}\right)^{2 / 5}+m+\sum_{\tau} n_{\tau}\right) \\
& =I^{\prime}+O\left(m^{3 / 5}\left(\sum_{\tau} n_{\tau}^{2}\right)^{2 / 5}+m+\sum_{\tau} n_{\tau}\right) .
\end{aligned}
$$


Taking expectation with respect to the random sample $\mathcal{R}$, and using the analysis of Clarkson and Shor [9] and the concavity of the function $x^{2 / 5}$, we obtain

$$
\begin{aligned}
I & =O(n)+O\left(m^{3 / 5} \cdot\left[\left(\frac{n}{r}\right)^{2} \cdot r\right]^{2 / 5}+m+n\right) \\
& =O\left(\frac{m^{3 / 5} n^{4 / 5}}{r^{2 / 5}}+m+n\right)=O\left(m^{3 / 5} P^{2 / 5}+m+n\right) .
\end{aligned}
$$

We now apply this bound to $\mathcal{C}$ and to the set $M$ of all $t_{\geq k}$ vertices incident to at least $k$ circles. Since the number of incidences is at least $k t_{\geq k}$, we obtain

$$
k t_{\geq k}=O\left(t_{\geq k}^{3 / 5} P^{2 / 5}+t_{\geq k}+n\right),
$$

from which the asserted bound on $t_{\geq k}$ follows readily.

Remark. An analogous bound to that derived in Lemma 4.9, which strengthens the bound in (4) that we have used earlier, can be established for arrangements of unit circles. Even though we do not need this variant, we include it here for the sake of completeness:

Lemma 4.10. Let $\mathcal{C}$ be a family of $n$ unit circles in the plane with $P$ pairs of intersecting circles. Then the number of points incident to at least $k$ circles is $O\left(P / k^{3}+n / k\right)$.

Proof. Using Székely's technique [18], it is easy to show that the number $I$ of incidences between the circles of $\mathcal{C}$ and a set $M$ of $m$ points satisfies

$$
I=O\left(m^{2 / 3} P^{1 / 3}+m+n\right) .
$$

Let $M$ be the set of all vertices of $\mathcal{A}(\mathcal{C})$ that are incident to at least $k$ circles of $\mathcal{C}$. Then $I \geq m k$, so we have $m k \leq c\left(m^{2 / 3} P^{1 / 3}+m+n\right)$, for an appropriate constant $c$, from which the claim follows readily.

Claim 4.11. Let $\mathcal{L}$ be a collection of $m>54 b$ lines in the plane. If $\mathcal{A}(\mathcal{L})$ does not contain a vertex (which may be at infinity) incident to more than $m / a$ lines, for any constant a satisfying $a>12 b$, then the number of distinct vertices of $\mathcal{A}(\mathcal{L})$ is at least $\mathrm{cm}^{2}$, for an appropriate constant $c$.

Proof. By applying a suitable projective transformation to the plane, we may assume that no two lines in $\mathcal{L}$ are parallel. Similar to (4) and the proof of Lemma 4.10, it has been shown in [19] (see also [14]) that, in an arrangement of $m$ lines, the number of vertices incident to at least $k$ lines is at most $b\left(m / k+m^{2} / k^{3}\right)$, for an appropriate absolute constant $b$. The number $Q$ of pairs of crossing lines is, by assumption, $\left(\begin{array}{c}m \\ 2\end{array}\right)$. Hence, denoting by $w_{k}$ (resp. $w_{\geq k}$ ) the number of vertices of $\mathcal{A}(\mathcal{L})$ incident to exactly (resp. at least) $k$ lines, and using an approach similar to the one in the proof of Theorem 4.5 we have, for a parameter $B$ that will be determined shortly,

$$
Q=\sum_{k=2}^{m / a}\left(\begin{array}{l}
k \\
2
\end{array}\right) w_{k}=\sum_{k=2}^{B-1}\left(\begin{array}{l}
k \\
2
\end{array}\right) w_{k}+\sum_{k=B}^{m / a}\left(\begin{array}{l}
k \\
2
\end{array}\right) w_{k}
$$




$$
\begin{aligned}
& \leq \frac{B}{2} \sum_{k=2}^{B-1} k w_{k}+\left(\begin{array}{l}
B \\
2
\end{array}\right) w_{\geq B}+\sum_{k=B+1}^{\sqrt{m}} k w_{\geq k}+\sum_{k=\sqrt{m}+1}^{m / a} k w_{\geq k} \\
& \leq \frac{B}{2} \sum_{k \geq 2} k w_{k}+\frac{b m^{2}}{B}+\sum_{k=B+1}^{\sqrt{m}} \frac{2 b m^{2}}{k^{2}}+\sum_{k=\sqrt{m}+1}^{m / a} 2 b m \\
& \leq \frac{B\left(E^{\prime}-m\right)}{2}+\frac{3 b m^{2}}{B}+\frac{2 b m^{2}}{a} \leq \frac{B\left(3 V^{\prime}+m\right)}{2}+\frac{3 b m^{2}}{B}+\frac{2 b m^{2}}{a},
\end{aligned}
$$

where $V^{\prime}$ and $E^{\prime}$ are the numbers of vertices and edges of the line arrangement, respectively. Hence, if we choose $a>12 b$ and $B=18 b$, we will have

$$
\frac{m^{2}}{6} \leq 9 b\left(3 V^{\prime}+m\right)
$$

and this implies the claim.

Claim 4.12. $\mathcal{A}(\mathcal{C})$ satisfies the following inequality:

$$
\beta n\left(q+n^{1 / 3}\right) \leq P \leq \sum_{k \geq 4}\left(\begin{array}{l}
k \\
2
\end{array}\right) t_{k} \leq 4 \sum_{k=4}^{\lambda}\left(\begin{array}{l}
k \\
2
\end{array}\right) t_{k},
$$

where

$$
\lambda=\max \left\{a q, c^{\prime}\left(P^{2 / 9}+n^{1 / 3}\right)\right\},
$$

for an appropriate constant $c^{\prime}$.

Proof. Let $v$ be a vertex of $\mathcal{A}(\mathcal{C})$ incident to $k>\lambda$ circles. Let $\mathcal{C}^{\prime}$ denote the subfamily of circles incident to $v$. Apply to the plane an inversion centered at $v$. All the circles in $\mathcal{C}^{\prime}$ are mapped into lines. No vertex (which may be at infinity) of this line arrangement is incident to more than $q$ lines, for otherwise $\mathcal{C}$ would contain a pencil of size larger than $q$. Since $k>a q$, Claim 4.11 implies that the line images of the circles of $\mathcal{C}^{\prime}$, and thus the circles of $\mathcal{C}^{\prime}$ themselves, intersect in at least $c k^{2}$ distinct points. Since $k \geq c^{\prime}\left(P^{2 / 9}+n^{1 / 3}\right)$, simple calculation shows that $t_{\geq \lambda}<\frac{1}{2} c \lambda^{2}<\frac{1}{2} c k^{2}$, so at least half of these intersection points are each incident to at most $\lambda$ circles. This implies that the number of pairs of circles meeting at high-degree vertices can be charged to twice the number of pairs of circles meeting at low-degree vertices. In other words, we have shown that

$$
\sum_{k>\lambda}\left(\begin{array}{l}
k \\
2
\end{array}\right) t_{k} \leq 3 \sum_{k \leq \lambda}\left(\begin{array}{l}
k \\
2
\end{array}\right) t_{k} .
$$

This readily implies the claim.

We next estimate the sum in Claim 4.12 using (5). That is, we have, for a parameter $B$ that will be determined shortly and for $\xi=(P / n)^{2 / 3}$,

$$
\beta n\left(q+n^{1 / 3}\right) \leq P \leq 4 \sum_{k=4}^{\lambda}\left(\begin{array}{l}
k \\
2
\end{array}\right) t_{k}=4 \sum_{k=4}^{B-1}\left(\begin{array}{l}
k \\
2
\end{array}\right) t_{k}+4 \sum_{k=B}^{\lambda}\left(\begin{array}{l}
k \\
2
\end{array}\right) t_{k}
$$




$$
\begin{aligned}
& \leq 2 B \sum_{k=4}^{B-1} k t_{k}+4\left(\begin{array}{l}
B \\
2
\end{array}\right) t_{\geq B}+4 \sum_{k=B+1}^{\xi} k t_{\geq k}+4 \sum_{k=\xi+1}^{\lambda} k t_{\geq k} \\
& \leq 2 B \sum_{k \geq 4} k t_{k}+\frac{4 b P}{B^{1 / 2}}+\sum_{k=B+1}^{\xi} \frac{8 b P}{k^{1.5}}+\sum_{k=\xi+1}^{\lambda} 8 b n \\
& \leq 2 B E+\frac{20 b P}{B^{1 / 2}}+8 \lambda b n .
\end{aligned}
$$

If $\lambda=a q$, i.e., $q=\Omega\left(P^{2 / 9}+n^{1 / 3}\right)$, then, choosing the constants $B, a$ and $\beta$ appropriately, we will have, using Theorem 3.14,

$$
P<4 B E \leq 16 B\left(f_{2}+2 f_{1}\right)=O\left(n^{1 / 2-\varepsilon} P^{1 / 2+\varepsilon}+n\right),
$$

for any $\varepsilon>0$. This implies that $P=O(n)$ and this will lead to a contradiction if we require $\beta$ to be sufficiently large.

Otherwise, for $q=O\left(P^{2 / 9}+n^{1 / 3}\right)$, we again can obtain

$$
P<4 B E+O(\lambda n) \leq 16 B\left(f_{2}+2 f_{1}\right)+O(\lambda n)=O\left(n^{1 / 2-\varepsilon} P^{1 / 2+\varepsilon}+n+n P^{2 / 9}+n^{4 / 3}\right),
$$

for any $\varepsilon>0$. This implies that $P=O\left(n^{4 / 3}\right)$, which again is a contradiction if $\beta$ is required to be sufficiently large.

Remark. Theorem 4.8 may fail if $P$ is not sufficiently large, as the following construction shows. Given parameters $n$ and $q$, draw $m=n / q$ concentric circles $C_{1}, \ldots, C_{m}$ and another circle $C$ that intersects each of them at two points; denote the intersection points of $C$ and $C_{i}$ by $a_{i}$ and $b_{i}$. Now replace each $C_{i}$ by a pencil of $q$ circles $C_{i 1}, \ldots, C_{i q}$ that pass through $a_{i}$ and $b_{i}$ and are sufficiently close to each other so that no pair of circles from different pencils intersect. Put $\mathcal{C}=\{C\} \cup\left\{C_{i j} \mid i \leq m, j \leq q\right\}$. This is a collection of $n+1$ circles whose union is connected, so that every vertex of their arrangement is incident to $q+1$ circles. In this case the size of the largest pencil is $q+1$ and the number of intersecting pairs of circles is $n+m\left(\begin{array}{l}q \\ 2\end{array}\right)=n(q+1) / 2$. This shows that Theorem 4.8 may fail if we do not require that the number of intersecting pairs of circles is substantially larger than $n$ times the size of the largest pencil.

\section{Intersecting Pairs and Vertices in Arrangements of Circles}

In this section we use the machinery developed in the preceding sections to obtain the following results, which relate the number of vertices of the arrangement to the number of intersecting pairs of circles, and which we believe to be of independent interest.

Theorem 5.1. Let $\mathcal{C}$ be a collection of $n$ unit circles, with $P$ intersecting pairs of circles. Then these circles intersect in at least $\beta P$ distinct points, for some constant $\beta>0$.

Proof. The proof proceeds by induction on $n$ and $P$. The claim clearly holds for any $n \geq 2$ and $P=1$ (for any $\beta<1$ ). We assume that it holds for all $n^{\prime}<n$ and all $P$, and for $n^{\prime}=n$ and $P^{\prime}<P$, and will show that it also holds for $n$ and $P$. 
Arguing as in the proof of Theorem 4.5, we claim that there exists a unit disk $\sigma$ which contains at least $a \xi^{2}$ distinct vertices of $\mathcal{A}(\mathcal{C})$, where $\xi$ is the maximum number of circles of $\mathcal{C}$ that intersect any unit disk, and where $a$ is an appropriate absolute constant. Indeed, if the condition in Lemma 4.6 is satisfied, then the claim is obvious. Otherwise, we choose a disk $\sigma$ for which $P_{\sigma}=\Omega\left(\xi^{2}\right)$, and repeat the analysis in the preceding section (without assuming anything about $t_{2}$ and $t_{3}$ ). Inequality (2) becomes

$$
\sum_{k \geq 2} k t_{k}^{(\sigma)}=O\left(t_{2}^{(\sigma)}+t_{3}^{(\sigma)}+f_{1}^{(\sigma)}+f_{2}^{(\sigma)}\right)=O\left(t_{2}^{(\sigma)}+t_{3}^{(\sigma)}+\xi^{4 / 3} \log \xi\right) .
$$

The bound for $P^{*}$ is derived exactly as above, and allows us to assume that $P^{*}<P_{\sigma} / 2$. This, combined with (7), yields, as above,

$$
\Omega\left(\xi^{2}\right)=P_{\sigma}=O\left(t_{2}^{(\sigma)}+t_{3}^{(\sigma)}+\xi^{4 / 3} \log \xi\right) .
$$

Hence, if $\xi$ is at least some appropriate and sufficiently large constant $\alpha$, then we have

$$
t_{2}^{(\sigma)}+t_{3}^{(\sigma)}=\Omega\left(\xi^{2}\right)
$$

which implies the claim. If $\xi<\alpha$, then, since $\mathcal{A}(\mathcal{C})$ contains at least one vertex, we can choose $\sigma$ to be a unit disk containing that vertex, and choose $a$ so that $1 \geq a \alpha^{2}$. Then in this case we also have a unit disk that contains at least $a \xi^{2}$ distinct vertices of $\mathcal{A}(\mathcal{C})$.

Remove from $\mathcal{C}$ all the $n_{\sigma}$ circles of $\mathcal{C}_{\sigma}$ (i.e., the circles that intersect $\sigma$ ), and let $\mathcal{C}^{\prime}$ be the resulting subset. Let $P^{\prime}$ denote the number of intersecting pairs of circles in $\mathcal{C}^{\prime}$. We have $P^{\prime}=P-P_{1}-P_{2}$, where $P_{1} \leq \xi^{2} / 2$ is the number of intersecting pairs of circles in $\mathcal{C}_{\sigma}$, and $P_{2}$ is the number of intersecting pairs $\left(C, C^{\prime}\right)$ of circles, with $C \in \mathcal{C}_{\sigma}$ and $C^{\prime} \in \mathcal{C}^{\prime}$. Note that any such circle intersects the disk $\sigma^{*}$ of radius 3 and concentric with $\sigma$. We can cover $\sigma^{*}$ by 19 unit disks, using a construction based on the hexagonal grid and similar to that shown in Fig. 7, and use the maximality of $\xi$ to conclude that the number of such circles $C^{\prime}$ is at most $19 \xi$. Hence, $P_{2} \leq 19 \xi^{2}$.

In other words, we have found $N \geq a \xi^{2} \geq 2 \beta\left(P_{1}+P_{2}\right)$ distinct vertices of $\mathcal{A}(\mathcal{C})$, for an appropriate choice of $\beta$. After removing $\mathcal{C}_{\sigma}$, we are left with a set $\mathcal{C}^{\prime}$ of $n^{\prime}<n$ circles, such that no vertex of $\mathcal{A}\left(\mathcal{C}^{\prime}\right)$ coincides with any of the vertices constructed so far. If $P^{\prime} \leq P / 2$, then $P_{1}+P_{2}=P-P^{\prime} \geq P / 2$, so we have shown that $\mathcal{A}(\mathcal{C})$ contains at least $2 \beta P / 2=\beta P$ distinct vertices. Otherwise, apply the induction hypothesis to $n^{\prime}$ and $P^{\prime}$, to obtain at least $\beta P^{\prime}$ new vertices of $\mathcal{A}(\mathcal{C})$. Hence, the number of distinct vertices of $\mathcal{A}(\mathcal{C})$ is at least

$$
\beta P^{\prime}+2 \beta\left(P_{1}+P_{2}\right) \geq \beta\left(P^{\prime}+P_{1}+P_{2}\right)=\beta P
$$

This establishes the induction step and thus completes the proof of the theorem.

Corollary 5.2. The number of distinct intersection points of $n$ unit circles whose centers lie inside a square of side length $d>1$ is at least $\Omega\left(n^{2} / d^{2}\right)$.

Proof. Assume $d$ to be an integer, and partition the given square into $d^{2}$ squares of side length 1 . Let $\mathcal{C}_{i}$ denote the subcollection of the given circles whose centers lie in the $i$ th smaller square, for $i=1, \ldots, d^{2}$. Every pair of circles in $\mathcal{C}_{i}$ intersect, so, by 
Theorem 5.1, these circles have $\Omega\left(n_{i}^{2}\right)$ distinct points of intersection, where $n_{i}=\left|\mathcal{C}_{i}\right|$. We sum these lower bounds over all families $\mathcal{C}_{i}$, and note that each intersection point can contribute to only $O(1)$ terms. Hence, the total number of intersection points of the given circles is $\Omega\left(\sum_{i} n_{i}^{2}\right)=\Omega\left(\left(\sum_{i} n_{i}\right)^{2} / d^{2}\right)=\Omega\left(n^{2} / d^{2}\right)$.

Remark. The corollary does not use the full "strength" of the theorem. It only requires the weaker result that if every pair of circles intersect, then they determine $\Omega\left(n^{2}\right)$ distinct intersection points.

\section{Corollary 5.3.}

(a) For a collection $\mathcal{C}$ of $n$ unit circles whose centers lie in a square of size $\leq c n^{1 / 2}$, for a sufficiently small constant $c$, there exists a point that is incident to only two or three circles.

(b) If the area of the union of the disks bounded by the circles of $\mathcal{C}$ is at most c'n, for a sufficiently small constant $c^{\prime}$, then there exists a point that is incident to only two or three circles.

Proof. In both cases it is easy to show that the number of pairs of intersecting circles in $\mathcal{C}$ is larger than $\beta n$, provided $c$ and $c^{\prime}$ are sufficiently small.

Theorem 5.4. Let $\mathcal{C}$ be a collection of $n$ circles of arbitrary radii in the plane with $P$ intersecting pairs. Let $q$ denote the largest size of a pencil in $\mathcal{C}$, and suppose that $P \geq \beta n\left(q+n^{1 / 3}\right)$, for a sufficiently large constant $\beta$. Then $\mathcal{A}(\mathcal{C})$ has $\Omega(P)$ distinct vertices.

Proof. Similar to the assertion of Claim 4.12, we have

$$
\beta n\left(q+n^{1 / 3}\right) \leq P \leq \sum_{k \geq 2}\left(\begin{array}{l}
k \\
2
\end{array}\right) t_{k} \leq 3 \sum_{k=2}^{\lambda}\left(\begin{array}{l}
k \\
2
\end{array}\right) t_{k},
$$

where $\lambda$ is as defined above. We estimate this sum using Lemma 4.9. That is, we have, for a parameter $B$ that will be determined shortly,

$$
\begin{aligned}
\beta n\left(q+n^{1 / 3}\right) & \leq P \leq 4 \sum_{k=2}^{\lambda}\left(\begin{array}{l}
k \\
2
\end{array}\right) t_{k}=4 \sum_{k=2}^{B-1}\left(\begin{array}{l}
k \\
2
\end{array}\right) t_{k}+4 \sum_{k=B}^{\lambda}\left(\begin{array}{l}
k \\
2
\end{array}\right) t_{k} \\
& \leq 2 B^{2} \sum_{k=2}^{B-1} t_{k}+4\left(\begin{array}{l}
B \\
2
\end{array}\right) t_{\geq B}+4 \sum_{k=B+1}^{(P / n)^{2 / 3}} k t_{\geq k}+4 \sum_{k=(P / n)^{2 / 3}+1}^{\lambda} k t_{\geq k} \\
& \leq 2 B^{2} \sum_{k \geq 2} t_{k}+\frac{4 c P}{B^{1 / 2}}+\sum_{k=B+1}^{(P / n)^{2 / 3}} \frac{8 c P}{k^{1.5}}+\sum_{k=(P / n)^{2 / 3}+1}^{\lambda} 8 c n \\
& \leq 2 B^{2} V+\frac{20 c P}{B^{1 / 2}}+8 c n \lambda .
\end{aligned}
$$


Hence, choosing the constants $B$ and $\beta$ appropriately, one can show, as in the preceding analysis, that

$$
\frac{P}{2} \leq 2 B^{2} V
$$

which establishes the claim.

Remark. The theorem may fail if we do not require $P$ to be significantly larger than $n q$, as the example given at the end of the preceding section demonstrates.

\section{References}

1. P. K. Agarwal and J. Erickson, Geometric range searching and its relatives, in Advances in Discrete and Computational Geometry (B. Chazelle, J. E. Goodman and R. Pollack, Eds.), Contemporary Mathematics, Vol. 223, American Mathematical Society, Providence, RI, 1999, pp. 1-56.

2. P. K. Agarwal and J. Matoušek, On range searching with semialgebraic sets, Discrete Comput. Geom. 11 (1994), 393-418.

3. A. Bezdek, On the density of dual circle coverings, Geom. Dedicata 33 (1990), 227-238.

4. A. Bezdek, On the intersection points of unit circles, Amer. Math. Monthly 99 (1992), 779-780.

5. A. Bezdek, Incidence problems for points and unit circles, in Paul Erdós and His Mathematics (A. Sali, M. Simonovits and V. T. Sós, Eds.), J. Bolyai Math. Soc., Budapest, 1999, pp. 33-36.

6. A. Bezdek, On unit circles which avoid all but two points of a given pointset, European J. Combin., to appear.

7. A. Bezdek, F. Fodor and I. Talata, A Sylvester-type theorem about circles, Discrete Math., to appear.

8. K. Clarkson, H. Edelsbrunner, L. Guibas, M. Sharir and E. Welzl, Combinatorial complexity bounds for arrangements of curves and spheres, Discrete Comput. Geom. 5 (1990), 99-160.

9. K. Clarkson and P. Shor, Applications of random sampling in computational geometry, II, Discrete Comput. Geom. 4 (1989), 387-421.

10. J. Eckhoff, Helly, Radon and Carathéodory type theorems, in Handbook of Convex Geometry, Vol. A (P. M. Gruber and J. M. Wills, Eds.), North-Holland, Amsterdam, 1993, pp. 389-448.

11. H. Edelsbrunner, Algorithms in Combinatorial Geometry, Springer-Verlag, New York, 1987.

12. M. J. Katz and M. Sharir, An expander-based approach to geometric optimization, SIAM J. Comput. 26 (1997), 1384-1408.

13. J. Pach, Finite point configurations, in Handbook of Discrete and Computational Geometry (J. E. Goodman and J. O'Rourke, Eds.), CRC Press, Boca Raton, FL, 1997, pp. 3-18.

14. J. Pach and P. K. Agarwal, Combinatorial Geometry, Wiley-Interscience, New York, 1995.

15. R. Pinchasi, Gallai-Sylvester theorem for pairwise-intersecting unit circles in the plane, Manuscript, 1999.

16. M. Sharir and P. K. Agarwal, Davenport-Schinzel Sequences and Their Geometric Applications, Cambridge University Press, New York, 1995.

17. J. Spencer, E. Szemerédi and W. T. Trotter, Unit distances in the Euclidean plane, in Graph Theory and Combinatorics (B. Bollobás, Ed.), Academic Press, New York, 1984, pp. 293-303.

18. L. Székely, Crossing numbers and hard Erdős problems in discrete geometry, Combin. Probab. Comput. 6 (1997), 353-358.

19. E. Szemerédi and W. Trotter, Jr., Extremal problems in discrete geometry, Combinatorica 3 (1983), 381392.

20. H. Tamaki and T. Tokuyama, How to cut pseudo-parabolas into segments, Discrete Comput. Geom. 19 (1998), 265-290.

Received June 26, 2000, and in revised form January 30, 2001. Online publication October 5, 2001. 